\title{
Blank shape optimization considering 3D space targets for external and internal boundaries in sheet metal forming processes
}

Hamidreza Gharehchahi

Islamic Azad University

Mohammad Javad Kazemzadeh-Parsi ( $\sim$ MJ.Kazemzadeh.Parsi@gmail.com )

Islamic Azad University

Ahmad Afsari

Islamic Azad University

Mehrdad Mohammadi

Islamic Azad University

\section{Research Article}

Keywords: Deep drawing, Shape optimization, Blank optimization, internal boundary, Finite element method

Posted Date: April 6th, 2021

DOI: https://doi.org/10.21203/rs.3.rs-168141/v1

License: (c) (i) This work is licensed under a Creative Commons Attribution 4.0 International License. Read Full License 


\title{
Blank shape optimization considering 3D space targets for external and internal boundaries in sheet metal forming processes
}

\author{
Hamidreza Gharehchahi, Mohammad Javad Kazemzadeh-Parsi ${ }^{*}$, Ahmad Afsari, Mehrdad \\ Mohammadi \\ Department of Mechanical Engineering, Shiraz Branch, Islamic Azad University, Shiraz, Iran. \\ *Corresponding author: kazemzadeh@iaushiraz.ac.ir
}

\begin{abstract}
The optimum design of an initial blank shape in sheet metal forming processes is an important step in many industries, especially automobile manufacturers because it reduces production costs and material waste. To the best of our knowledge, no research has been conducted on the blank shape designs based on 3D space target contours. Moreover, the present study considers parts with internal boundaries and optimum design of the internal boundary, which are among the innovations of this research. By following the iterative simulation-based optimization process, a special updating algorithm was proposed to modify the blank geometry in each iteration and reach the optimum shape. The sheet forming was severely nonlinear, due to plastic behavior, large deformations, and frictional contact surfaces. Therefore, the updating formula should be robust enough to be insensitive to the initial guess for the blank. To evaluate the proposed updating formula, some numerical examples were solved and the results were presented. Finally, the robustness of the proposed algorithm was investigated in these numerical experiences, by considering different geometries, target contours, internal boundaries, and initial guesses. The present study reveals that the proposed algorithm can be effectively used to solve blank optimization problems for the deep drawing process.
\end{abstract}

Keywords: Deep drawing; Shape optimization; Blank optimization; internal boundary; Finite element method

\section{Introduction}

Deep drawing is a useful sheet metal forming process for shaping flat blanks into cup-like forms. The process begins by cutting the blank with a specific geometry and placing it on the die. The plunging of the punch into the die drives the blank in, forming it into the shape of the die. Often, the part of the blank that remains outside does not develop a favorable shape and should be recut. The second cutting process creates two main issues of increasing wastes and involving complexities that can raise the production cost. Therefore, production costs remain 
lower if additional cutting can be avoided or at least minimized. An optimum blank geometry can realize this objective. Blank optimization refers to selecting an initial blank geometry that develops the desired shape after deep drawing without or with minimal need for additional cutting. Regarding the time-consuming and costly nature of the trial and error-based blank optimization methods, researchers have attempted to use numerical approaches to designing an optimum blank. The use of blanks with optimum geometries offers many advantages in deep drawings, such as reducing the production cost, improving the process quality, thickness distribution, and formability of the part, minimizing forming defects such as wrinkling and rupture, and decreasing the number of trial and error steps in the product development process. The numerical simulation of the sheet metal forming process is integral to the study of the feasibility of production by deep drawing and the initial design of a new part with complex three-dimensional geometry.

So far, various optimization algorithms have been proposed to design blanks, the most notable algorithms of which can be classified into four groups, namely the slip line field [1-9], geometric mapping [10-13], inverse approach [14-24], and numerical simulation-based iterative methods [25-35]. Meanwhile, methods of the fourth category have been widely used by researchers, due to their higher simulation accuracy and more capacity to solve complex problems. The iterative methods are capable of simulating complex geometries, large deformations, complex material behavior models, complex friction models, complex contact models, dynamic behaviors, and other factors with high accuracy. However, demanding a large volume of calculations is the main drawback of numerical simulation-based iterative methods. Developing efficient optimization algorithms can reduce the number of iterations and computational complexity. The present study aims to propose a new, efficient, iteration-based algorithm and implement it by a finite element numerical method to resolve some of the issues related to such methods. Given that the proposed algorithm is classified as an iterative method and is based on numerical simulation, studies on similar algorithms are reviewed in the following paragraph.

In 1985, Toh et al., [25] was one of the earliest studies conducted on the application of numerical simulation-based iterative methods for blank optimization in deep drawing. The authors relied on a finite element method in the numerical simulation of the deep drawing process, using a geometric correction algorithm based on the material flow. The main shortcoming of this method is that material flow patterns are not a reliable reference for developing the geometric correction algorithm, due to the nonlinear behavior prevalent in deep drawing. Further, this method fails to be converged when the initial guess is far from the 
optimum blank. In 1997, Chung et al. [26] developed a sequential design method for drawing the ideal forming theory, by using finite element analysis and experimental investigation. They applied the method to design blanks from a severely anisotropic aluminium sheet, aiming to minimize corners. In 1999, Park et al. [28] proposed a blank design method by combining the ideal forming method with a deformation path iteration method based on the finite element analysis. First, a test blank was prepared based on the ideal forming theory. Then, the optimum blank was obtained by the iterative deformation path iteration method. Although this method is applicable in cases where the depth of drawing and sheet deformation are limited, it reduces the convergence rate in case of a high depth of drawing and severe deformation. In 2002, Pegada et al. [29], used the iterative design procedure in an algorithm to detect optimum blank contours, considering anisotropy and friction in the deep drawing of an aluminium cup. In the same year, Shim et al. [30] applied the iterative sensitivity method for blank optimization, in which sensitivity was calculated by the finite element analysis of deformation using both main and offset blanks. In 2003, Son et al. [31] proposed the initial velocity of the boundary nodes (INOV) method, in which the ratio of the initial velocity boundary nodes to the entire path was used in the optimized blank algorithm. In 2008, Vafaeesefat [32] utilized iteration and the finite element simulation for blank optimization. The blank correction algorithm was based on the projection of the target contour over the deformed blank (boundary projection method). In the same year, Azaouzi et al. [33] introduced an initial blank estimation method based on the one-step inverse approach and optimized the blank geometry by drawing iterations and integrating heuristic optimization algorithms into the finite element analysis. In 2009, Hammami et al. [34] developed an iteration-based method with an experimental initial blank using the Push-Pull design optimization technique. They also studied the effect of initial anisotropy in obtaining the initial blank shape [35]. In 2012, Fazli et al. [27] proposed a novel iteration-based blank optimization technique and improved the method employed by Son et al. [31], recommending to correct the initial blank based on the difference between the final shape and the target shape to adjust boundary nodes. Comparing the results with INOV and PushPull reveals that the final products of the algorithms are practically similar with no apparent distinction and the proposed method is more efficient.

However, the above algorithms have faced the issue of the inefficiency of the algorithm when the initial guess is far from the optimum blank shape. In other words, the algorithms have failed to be converged if a near-optimum initial guess is not provided. For example, heuristic methods were employed to tackle this problem and find a suitable initial guess to be submitted to the optimization algorithm [27]. Such techniques depend on the problem, which limits the 
generality of the method and its application in different problems. As a result, the main objective of the present study is to achieve an algorithm that can solve the optimization problem, regardless of the initial guess. For this purpose, the deep drawing process was numerically simulated, and then, the proposed algorithm was implemented by the finite element method using the commercial software ABAQUS. Further, the optimization algorithm was programmed by Python. Finally, five numerical examples were solved based on the method to indicate the ability of the proposed algorithm to be converged on a favorable solution, regardless of the initial guess.

\section{Iterative simulation-based shape optimization}

Shape optimization problems are classified as variable-domain problems because the geometry of the problem domain should be determined as a part of the solution process. In these problems, the unknown boundaries are often parameterized for searching in a space with a limited number of dimensions, which is performed by selecting some key points on the boundary and connecting them together. Founding the coordinates of the key points consolidates unknown boundaries. Figure 1 illustrates a schematic representation of a blank shape optimization problem in the sheet metal forming process.

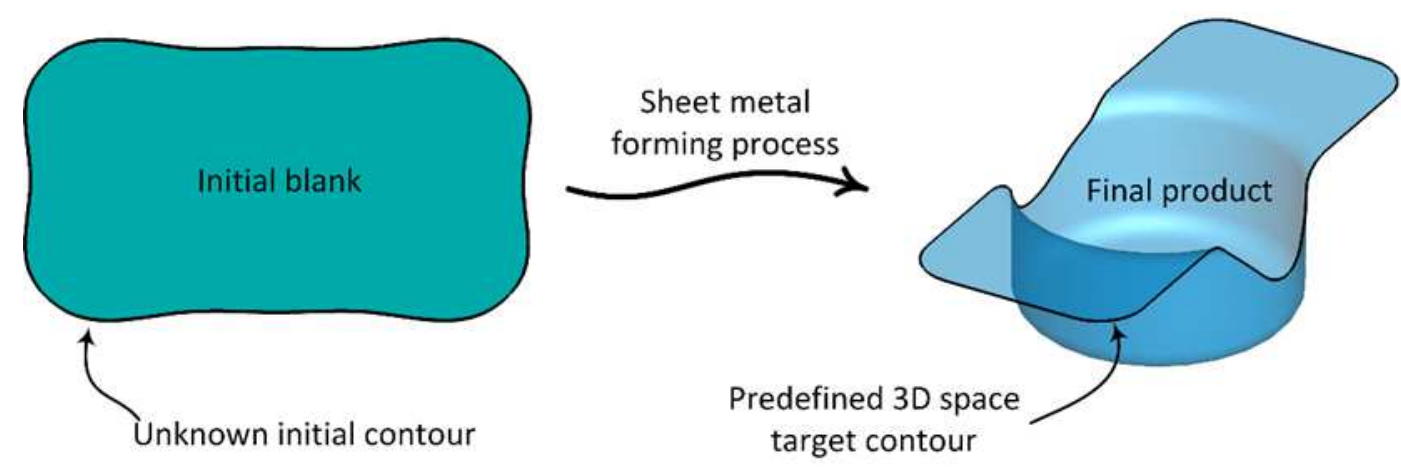

Figure 1: Initial blank shape optimization problem with 3D space target contour

As observed, the initial blank (undeformed part) and the final product (deformed part) are shown. The problem is to find the geometry of the initial contour such that the final contour matches the specified target after deformation.

Solving the shape optimization problems by simulation-based optimization methods consists of the following steps. First, an initial guess is considered for the blank shape (initial contour). Then, the sheet metal forming process is simulated based on the initial guess to obtain the final shape of the part (final contour). The final contour does not match the target contour at this 
stage. As a result, the mismatch between the final and target contours can be quantified by defining a shape error parameter. The geometry of the initial contour is then modified and the forming process is simulated again. The algorithm continues and reduces the shape error iteratively until the error drops below a set level and consequently, the process is stopped. Figure 2 provides the general flow chart of the simulation-based optimization steps.

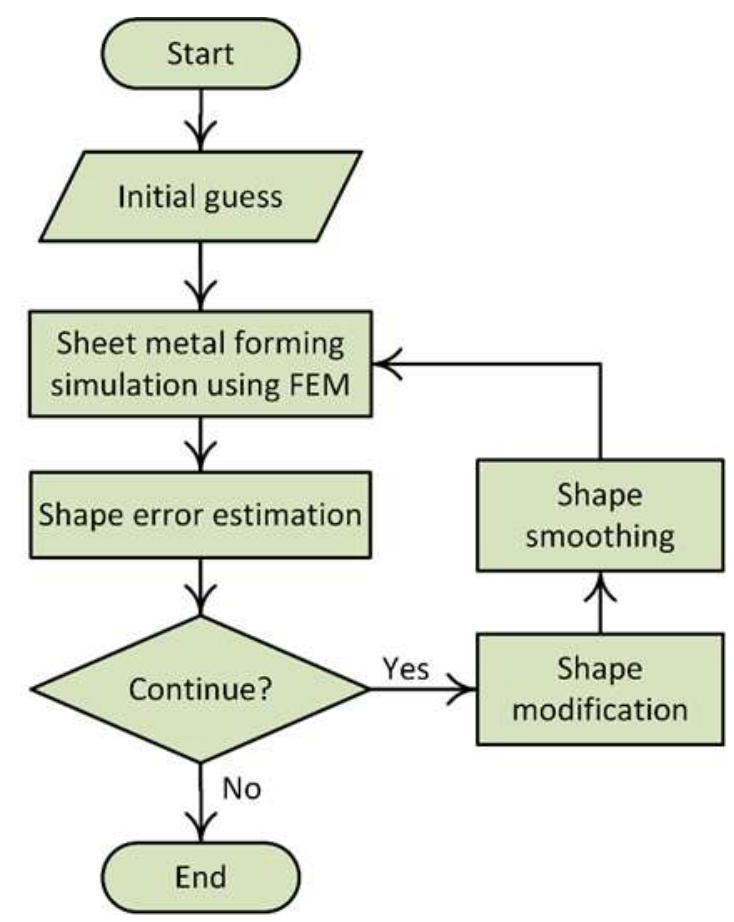

Figure 2: The flow chart of blank optimization by the iterative method based on numerical simulation

\section{Shape error estimation}

The objective of the optimum design problem is to minimize the difference between the final contour and the target. To this aim, the target shape is represented using a narrow ribbon in the 3D space. Figure 3(a) demonstrates a schematic representation of the initial contour, final contour, and the target ribbon. It is worth noting that CAD software facilitates generating such a target ribbon. In the first iteration, an initial guess should be considered for the blank geometry and the final contour would obviously be far from the target ribbon in this stage. Figure 3(b) shows the flow path of the material points that start from the initial contour and ended at the final contour. 


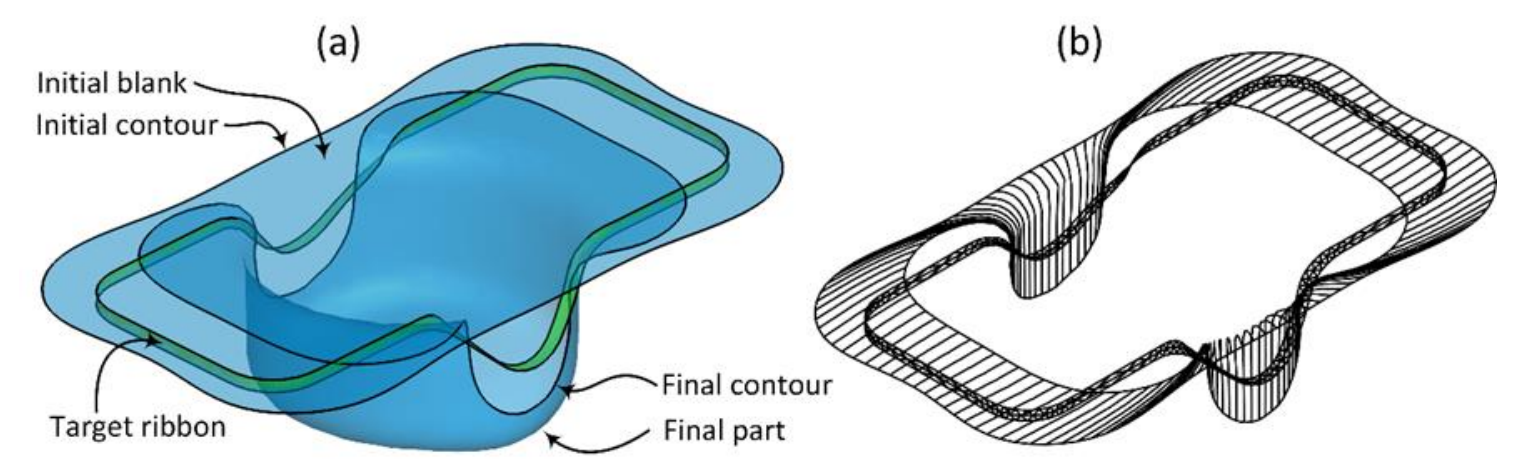

Figure 3: (a) A schematic representation of blank shape optimization problem with 3D space target; (b)

The flow path of the material points from initial contour to final contour in the sheet metal forming process

Some material points pass the target while some others fail to reach the target. As shown in Figure 2, the optimization procedure should be followed to capture the optimum initial contour. The optimization procedure has two important steps of shape error estimation and modification formula. The two following sections explain these two steps.

For each point of the initial contour, the shape error is defined as the shortest distance between the corresponding point on the final contour and the target ribbon. In Figure 4, two representative points $\mathrm{A}$ and $\mathrm{B}$ are shown on the initial contour and the corresponding points on the final contour are shown by A' and B', respectively.

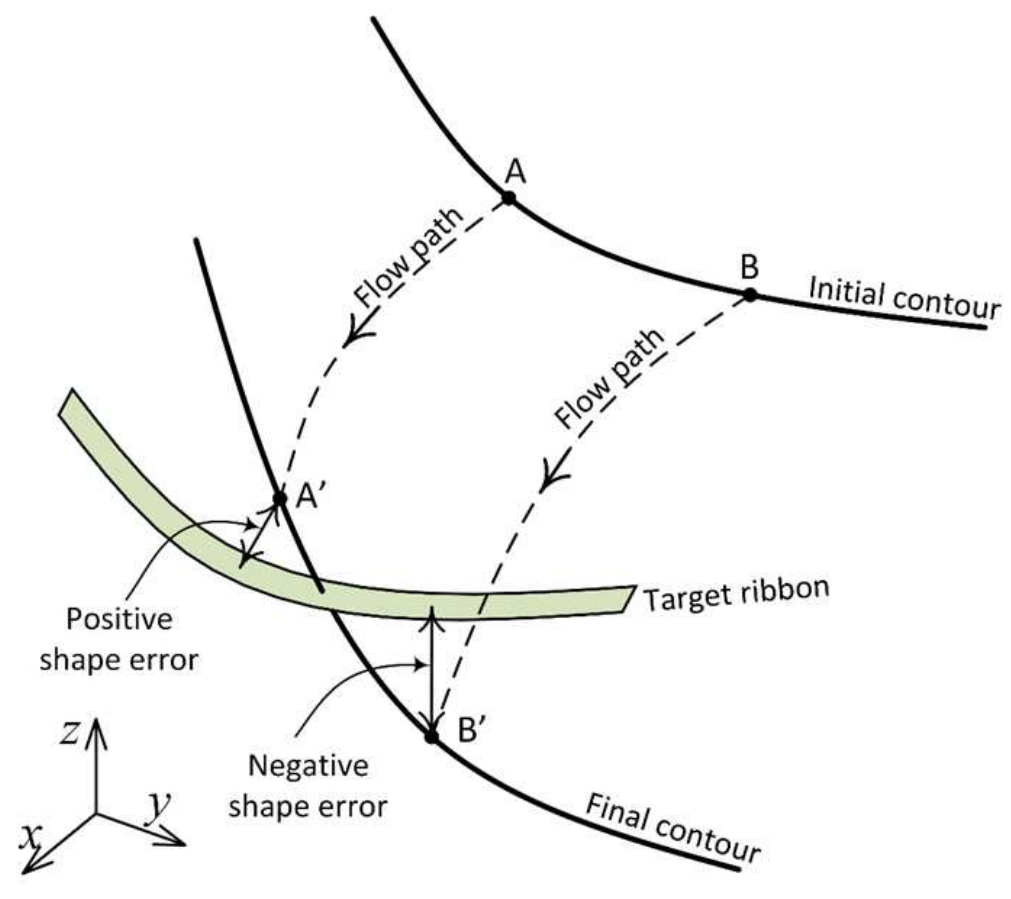

Figure 4: Definition of the shape error 
The shortest distance between final points $\mathrm{A}^{\prime}$ and $\mathrm{B}^{\prime}$ and the target ribbon is considered here as the absolute value of the shape error. If the flow path fails to pass the target ribbon, the shape error is considered as positive and if the flow path passes the target ribbon, the shape error is considered as negative. The shape error will be used in the modification formula to update the shape of the initial blank.

\section{Shape modification}

In this section, a robust and simple shape modification formula is presented to update the location of the key points on the initial contour in each step. As illustrated in Figure 5, two representative points $\mathrm{A}$ and $\mathrm{B}$ on the initial contour should be considered to explain the modification formula.

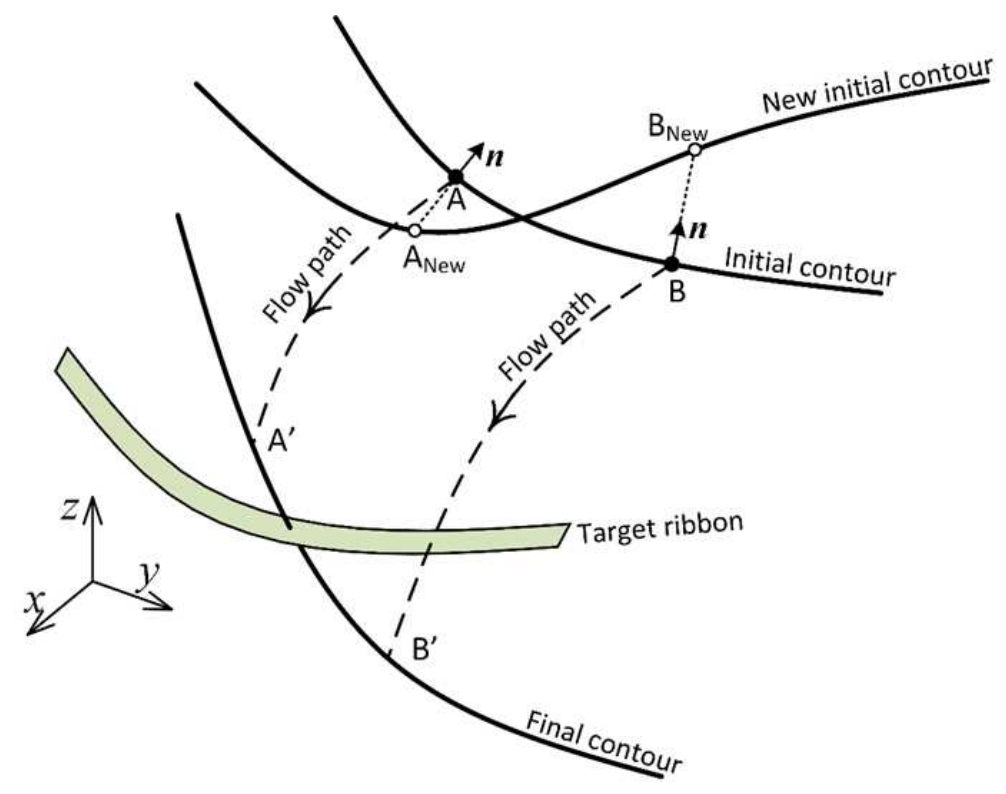

Figure 5: Modification of the initial contour

The shape error corresponding to each boundary point is obtained as explained in section 3 . For each point of the initial contour, consider unit vector $\mathrm{n}$ normal and outward with respect to the initial contour. The unit vectors corresponding to two representative points A and B are shown in Figure 5. The coordinate updating formula of the points of the initial contour is proposed as follow:

$$
\boldsymbol{P}_{\text {New }}=\boldsymbol{P}-\alpha e \boldsymbol{n}
$$

where $\mathrm{P}$ and $\mathrm{P}_{\mathrm{New}}$ represent the current and updated coordinate vectors of the points on the initial contour, respectively, e indicates the shape error, as explained in the previous section (see Figure 4), and $\alpha$ is the under-relaxation factor. This factor should be selected empirically 
less than unity to increase the stability of the optimization iterative procedure, due to the nonlinear behavior of the sheet metal forming process.

Figure 5 also displays a schematic representation of the updating formula. As shown, the new coordinates corresponding to two representative points A and B are shown with hollow circles. The point A has a positive shape error (see Figure 4). In other words, the corresponding point $A^{\prime}$ on the final contour failed to reach the target ribbon, meaning that there are some excessive materials near this point and these redundant materials should be removed from this region in the next iteration. Therefore, point A should move in the opposite direction of the normal vector $\mathrm{n}$ in this point.

Further, a similar procedure can be followed for point B. This point passes the target ribbon during the sheet forming process and has a negative shape error (see Figure 4), implying that there is less material near this point and the updating procedure should add some materials in this region. Therefore, point $\mathrm{B}$ should move along the unit vector $\mathrm{n}$. The updating formula given in Eq. (1) can be applied for all key-points on the initial contour to obtain the new initial contour, as shown schematically in Figure 5.

\section{Shape smoothing}

The sheet metal forming is a highly nonlinear process, due to complex material and geometric behaviors. Thus, the shape modification algorithm may show some instabilities. The under-relaxation factor, $\alpha$, which was introduced in the previous section, is a controlling parameter that tries to increase the stability of the optimization process. The under-relaxation factor also seeks to limit large movements of the initial contours in successive iterations. The other source of instability is zigzag patterns that may appear in the shape modification step. To remove the zigzag patterns, a smoothing step is considered in the optimization procedure (see Figure 2).

To explain the smoothing step, consider Figure 6. As shown, a part of the modified initial blank is shown schematically with a thick solid line. By considering two adjacent points $\boldsymbol{P}_{i}$ and $\boldsymbol{P}_{i+1}$, the directions of the angles $\theta_{i}$ and $\theta_{i+1}$ are addressed as an indicator to show whether a zigzag pattern appears at this part of the contour. If both angles $\theta_{i}$ and $\theta_{i+1}$ are in the same direction (both $\mathrm{CW}$ and both $\mathrm{CCW}$ ), no zigzag pattern has appeared in this part of the contour. Otherwise, this part of the contour is considered as a zigzag pattern. In this case, the following smoothing formula is used to update the location of boundary points $\boldsymbol{P}_{i}$ and $\boldsymbol{P}_{i+1}$.

$$
\boldsymbol{P}_{i}^{\text {smooth }}=(1-\beta) \boldsymbol{P}_{i}+\frac{\beta}{2}\left(\boldsymbol{P}_{i-1}+\boldsymbol{P}_{i+1}\right)
$$




$$
\boldsymbol{P}_{i+1}^{\text {smooth }}=(1-\beta) \boldsymbol{P}_{i+1}+\frac{\beta}{2}\left(\boldsymbol{P}_{i}+\boldsymbol{P}_{i+2}\right)
$$

where $\beta$ represents a smoothing parameter. It should be noted that value $\beta=0$ means no smoothing while $\beta=1$ leads to the movement of each point to the midpoint of left and right adjacent points. Figure 6 depicts the smoothed initial contour with a thick dashed line. This smoothing procedure should be applied in all parts of the modified initial contours showing a zigzag pattern. However, selecting a relatively small value for the smoothing parameter requires implementing the smoothing operator several times to whole points of the initial contour.

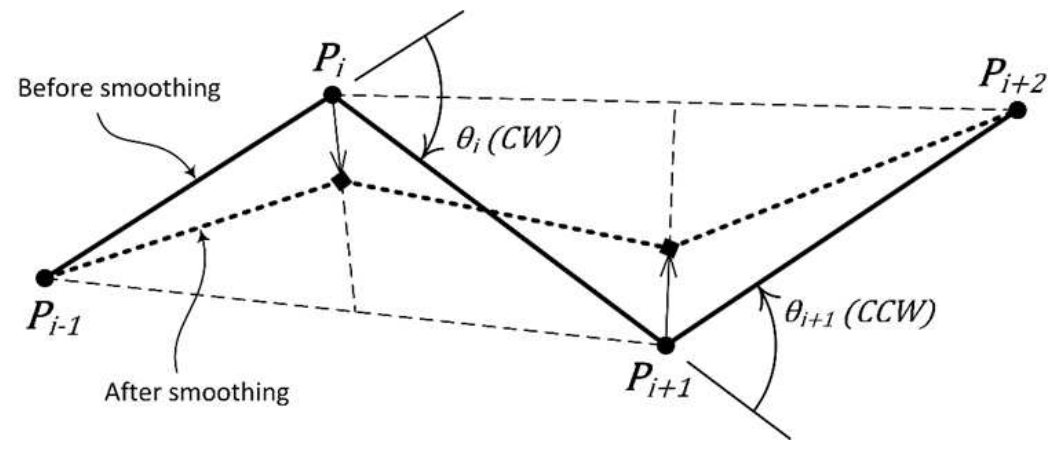

Figure 6: Smoothing the zigzag parts of the boundary

\section{Numerical examples}

In the following, some numerical examples were solved and the results were analyzed to evaluate the proposed algorithm. The sheet metal forming process was numerically simulated by using the finite element method and the commercial software, ABAQUS. Additionally, deformations were assumed large, and nonlinear geometric effects were taken into account. The frictional contact constraint was considered between all connected surfaces, and the friction Coulomb model was applied by the penalty method. The material was assumed to have an elastic-plastic behavior, regardless of strain rate and temperature effects. A Python program was developed in ABAQUS software to implement the whole optimization process.

In all examples, the sheet metal was assumed to have a thickness of $0.85 \mathrm{~mm}$. The stress-strain behavior in the plastic region was characterized by using a power-law relation as follows.

$$
\sigma_{p}=k\left(\varepsilon_{0}+\epsilon_{p}\right)^{n}
$$

where $\epsilon_{p}$ and $\sigma_{p}$ represent the plastic strain and stress, respectively. Table 1 presents the elastic properties and the coefficients of plastic behavior [27]. The die, punch, and plate holder were modeled as rigid parts. The only deformable part was the plate and the four-node shell elements were used to simulate the sheet metal forming process. 
Table 1.

Mechanical properties of the blank [27].

\begin{tabular}{ccc}
\hline Parameter & Symbol & Value \\
\hline Young modulus & $E$ & $200 \mathrm{GPa}$ \\
Poisson ratio & $v$ & 0.3 \\
Stress constant & $k$ & $514 \mathrm{MPa}$ \\
Strain constant & $\epsilon_{0}$ & 0.001 \\
Strain exponent & $n$ & 0.2 \\
Elastic limit & $S_{y}$ & $129.11 \mathrm{MPa}$ \\
\hline
\end{tabular}

\subsection{Example 1: L-shaped cup}

For the first example, the deep drawing of an L-shaped cup is considered [27]. Figure 7 displays all geometric details and dimensions (in millimetres) of the tools. The depth of drawing was considered $20 \mathrm{~mm}$ and a $9800 \mathrm{~N}$ blank holder force was applied, which remained constant throughout the process. Table 2 reports the coefficients of friction between contacting surfaces [27]. The target contour is considered such that a $2 \mathrm{~mm}$ uniform flange was formed all around the part.
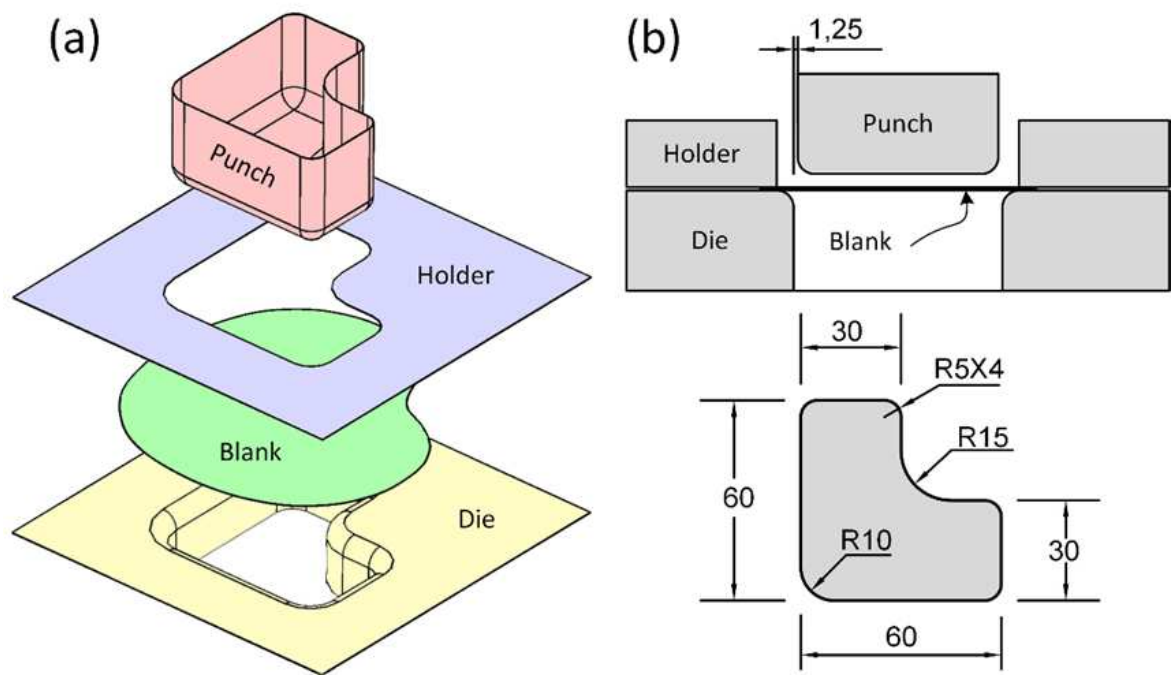

Figure 7: (a) Sheet metal forming tools assembly; (b) Dimensions of the tools for example 1 
Table 2.

Coefficients of friction between contacting surfaces [27].

\begin{tabular}{lcc}
\hline Contacting surfaces & $\begin{array}{c}\text { Coefficients of friction } \\
\text { for example 1 }\end{array}$ & $\begin{array}{c}\text { Coefficients of friction } \\
\text { for example 2 }\end{array}$ \\
\hline Blank and punch & 0.24 & 0.10 \\
Blank and die & 0.12 & 0.05 \\
Blank and plate holder & 0.12 & 0.05 \\
\hline
\end{tabular}

A regular hexagonal shape was considered as the initial guess for the initial contour. Figure 8 depicts the first four steps of the optimization process. The selected initial guess is far from the optimum shape and, therefore, the final contour is also far from the target. The initial contour was modified iteratively in subsequent steps and the final contour converged to the target shape. The proposed optimization procedure could capture the optimum shape only in four modification steps. In other words, only five numerical simulations were required to capture the optimum shape, regardless of the initial guess. The small number of iterations is an advantage of the proposed method as few iterations lead to lower computational costs and high efficiency. After four iterations, the maximum shape error was at $0.45 \mathrm{~mm}$ and the average shape error across all key points was at $0.11 \mathrm{~mm}$.
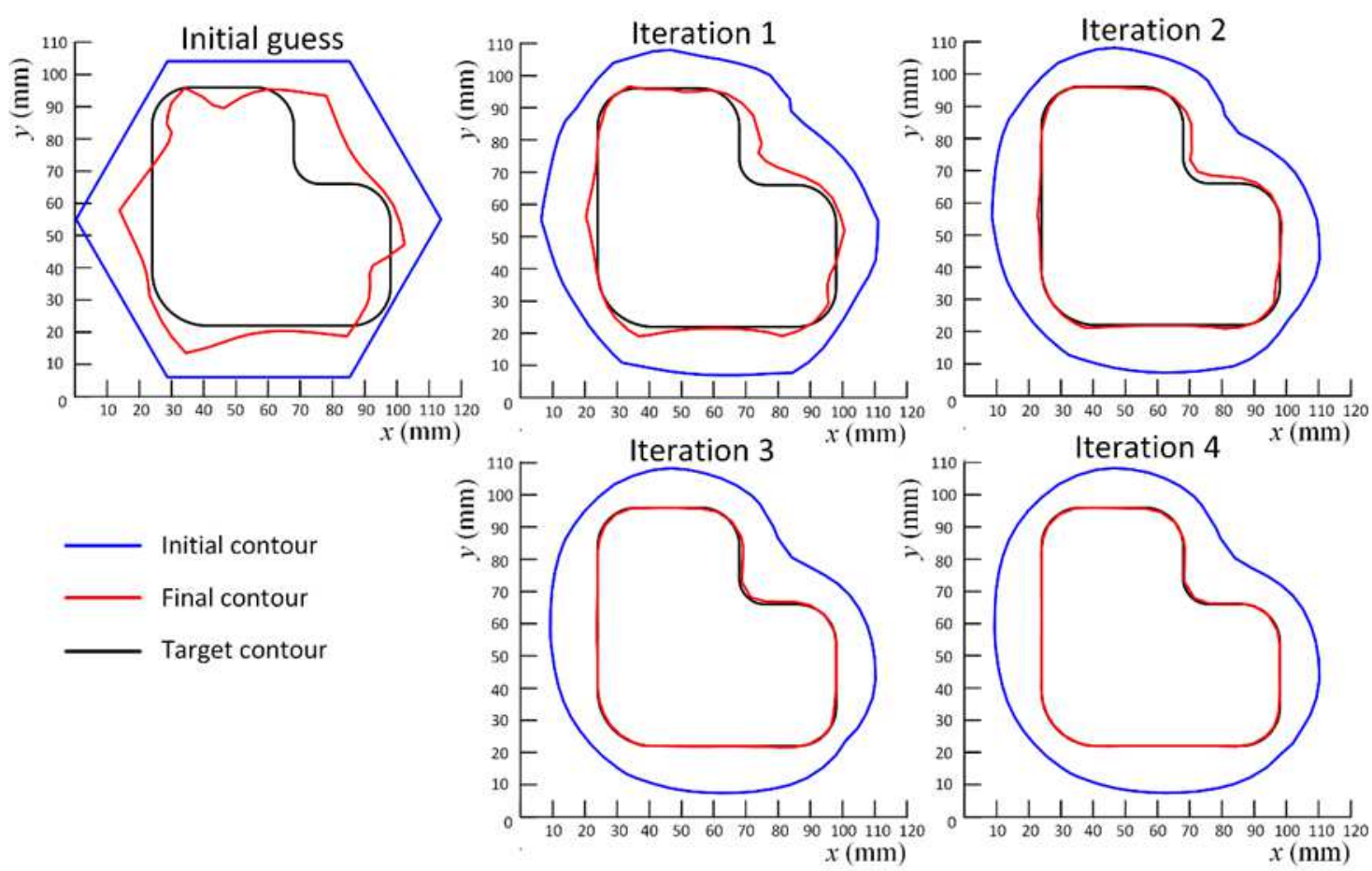

Figure 8: Initial and final contours for the first four iterations starting from a hexagonal initial guess for example 1. 
To investigate the effect of initial guess, two different initial guesses were selected, the results of which are given in Figure 9. Figs. 9(a) and (b) show the initial and final contours starting from the square and circular initial guesses, respectively. In both cases, the optimum shape is captured in four iterations. Figure 10 compares the optimum initial blanks, which are obtained from different initial guesses and the results given in Reference [27]. As observed, the selected initial guesses failed to affect the proposed algorithm, indicating the high accuracy and consistency of the results.
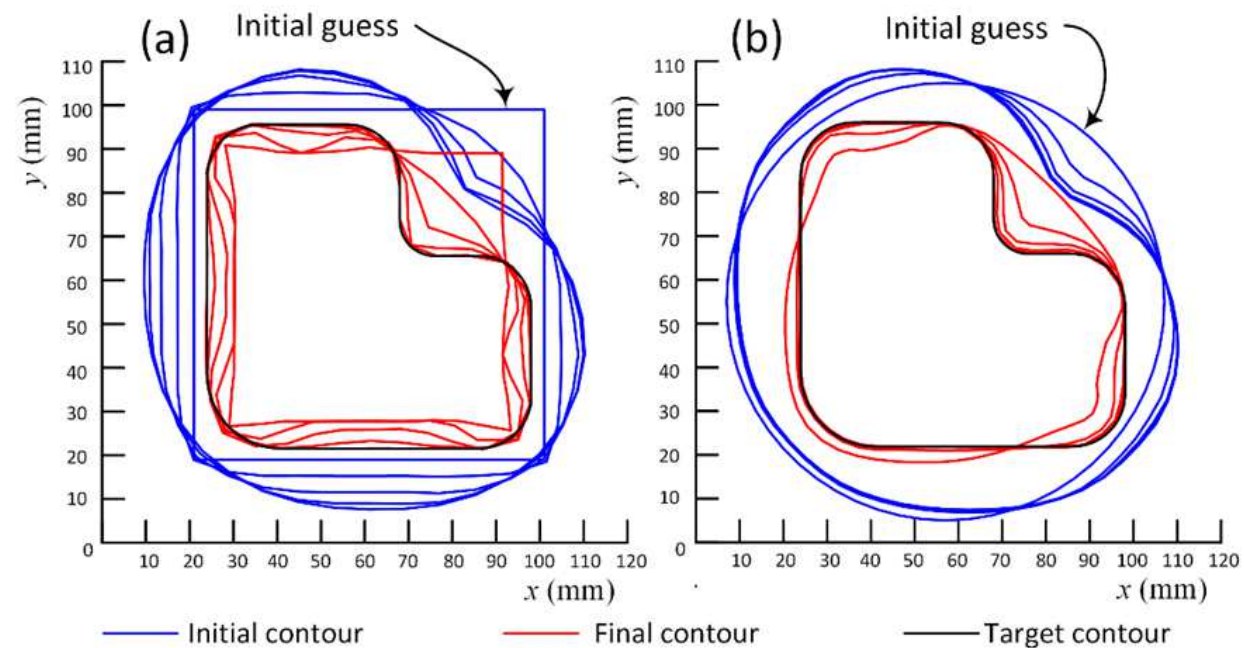

Figure 9: Initial and final contours for successive iteration of example 1; (a) starting from a squared initial guess; (b) starting from a circular initial guess.

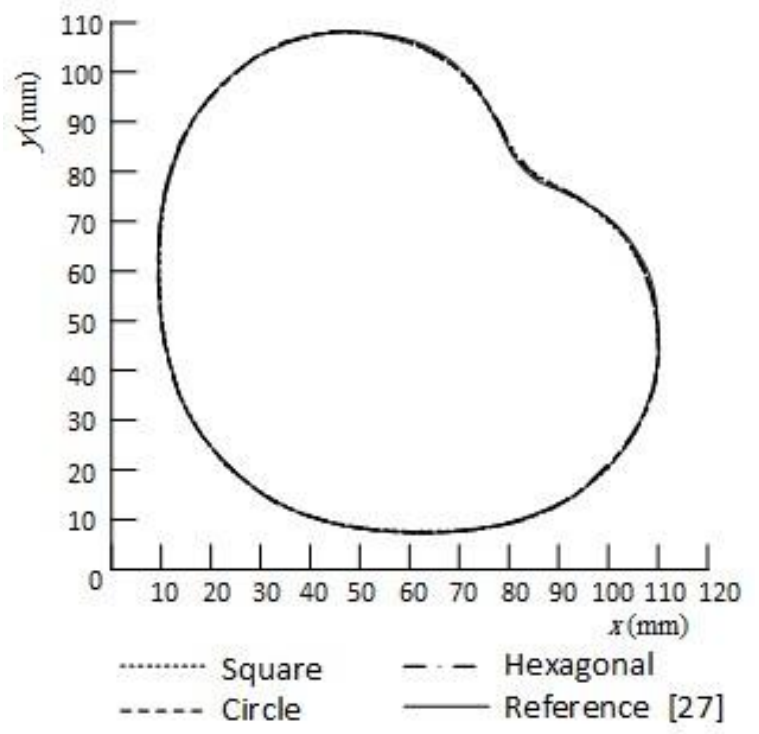

Figure 10: The optimum blanks obtained using three different initial guesses and comparing with the shape given in Reference [27]. 


\subsection{Example 2: two-level pan}

In this example, a deep drawing problem with two-level die and punch was considered and two different drawing depths were obtained [27]. The proposed algorithm was used to solve this problem with three different initial guesses. Figure 11 shows the geometrical dimensions of the die, blank holder, and the punch. All dimensions shown in Figure 11 are in millimeters.

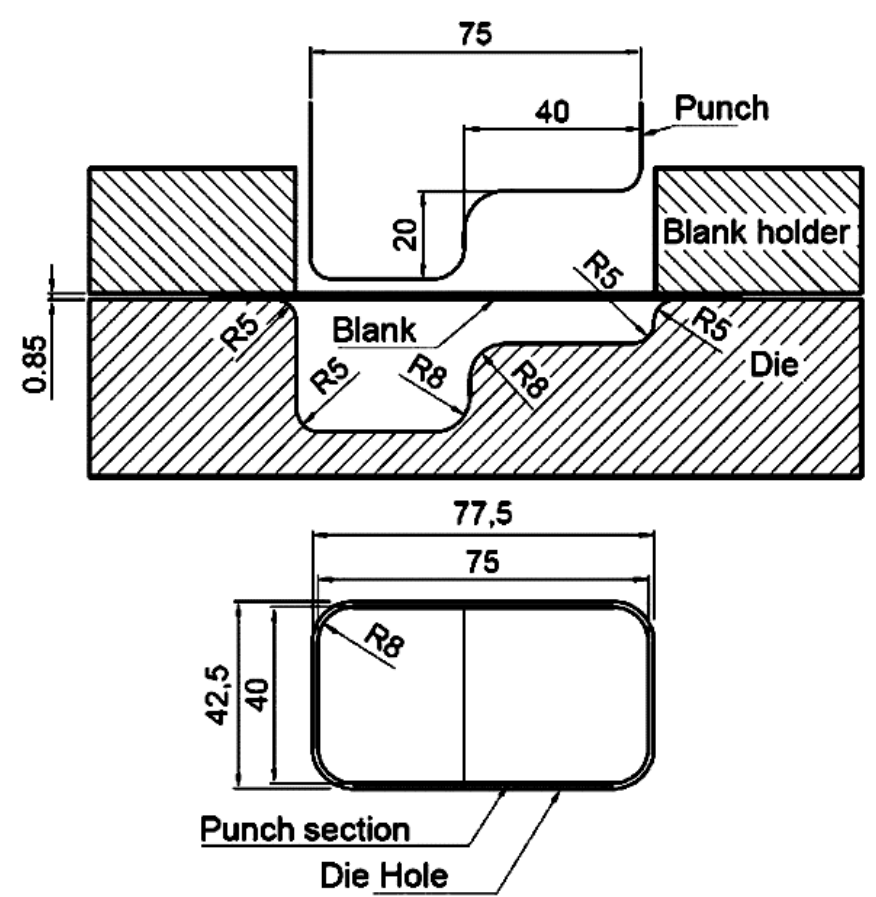

Figure 11: Dimensions of the tools for example 2.

The punch stroke was $30 \mathrm{~mm}$ and the target contour was a uniform edge of $1.75 \mathrm{~mm}$ width across the workpiece. A $17800 \mathrm{~N}$ blank holder force was also considered, which was assumed to remain constant during the process. Table 2 reports the coefficients of friction between all surfaces. Further, the under-relaxation factor was assumed at $0.6 \mathrm{~mm}$.

Figure 12(a) displays the three different initial guesses: circular, rectangular, and zigzag shape. As mentioned earlier, the number of iterations to reach the required accuracy increased to five iterations in this problem, due to the more complex behavior resulting from the two-level punch. The optimization problem was solved using different initial guesses and the optimum initial blank was obtained in each case after five iterations (see Figure 12(b)). The final contours are also plotted in Figure 12(c) and compared with the target contour. The final contours were aligned with the target contour for all initial guesses. In other words, using different and farinitial guesses had little impact on the final optimum blank configuration. 
Although the initial guesses were considerably different from the optimum in this example, the proposed algorithm managed to achieve the optimum in less than five iterations.

(a) Initial guesses

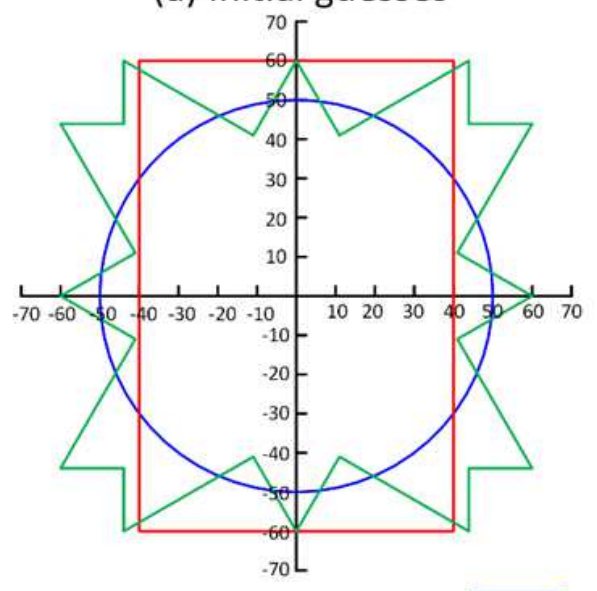

(b) Initial contoures

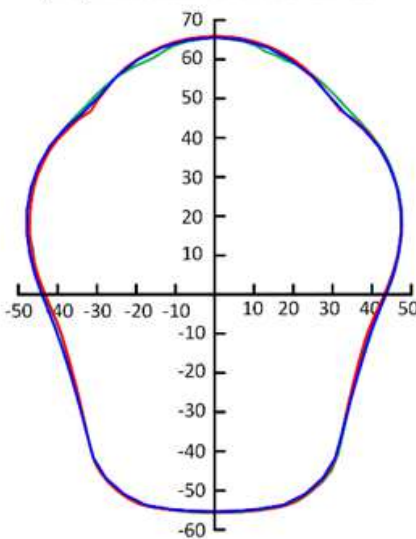

$\begin{array}{lll}- & \text { Circle } & \text { Zigzag } \\ & \text { Rectangle } & \text { Target }\end{array}$

(c) final contoures

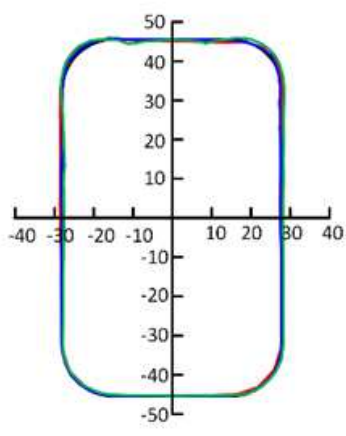

Figure 12: Results of example 2; (a) Three different initial guesses for the initial blank; (b) The optimum initial blank obtained with three different initial guesses; (c) The final contours obtained from three different initial guesses.

\subsection{Example 3: Symmetric flange}

It should be noted that the target contours are flat and are completely located in a plane in the previous two examples. However, the targets are 3D space contours in the present and the next examples. In the current example, the punch and the die are circular and the edges are rounded. The geometric details of the tools are given in Figure 13.

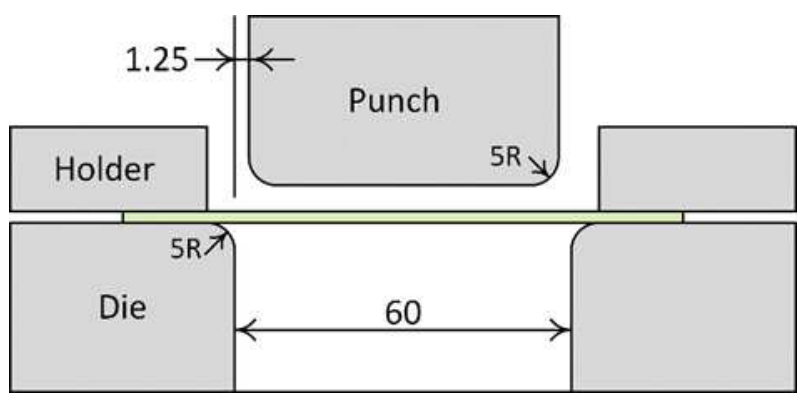

Figure 13: The geometric details of the tools for example 3.

The material, coefficients of friction, and blank holder force are similar to example 1 . The geometric details of the target shape are also provided in Figure 14. Figure 14(a) shows the front and top view of the target final product while Figure 14(b) demonstrates the 3D view of the target product. As explained before, the target shape is defined using the target ribbon, 
which can be generated simply in CAD software. The target ribbon is then used in the optimization procedure to compute the shape error and update the initial contour. Figure 14(c) provides the target contour for the present example.
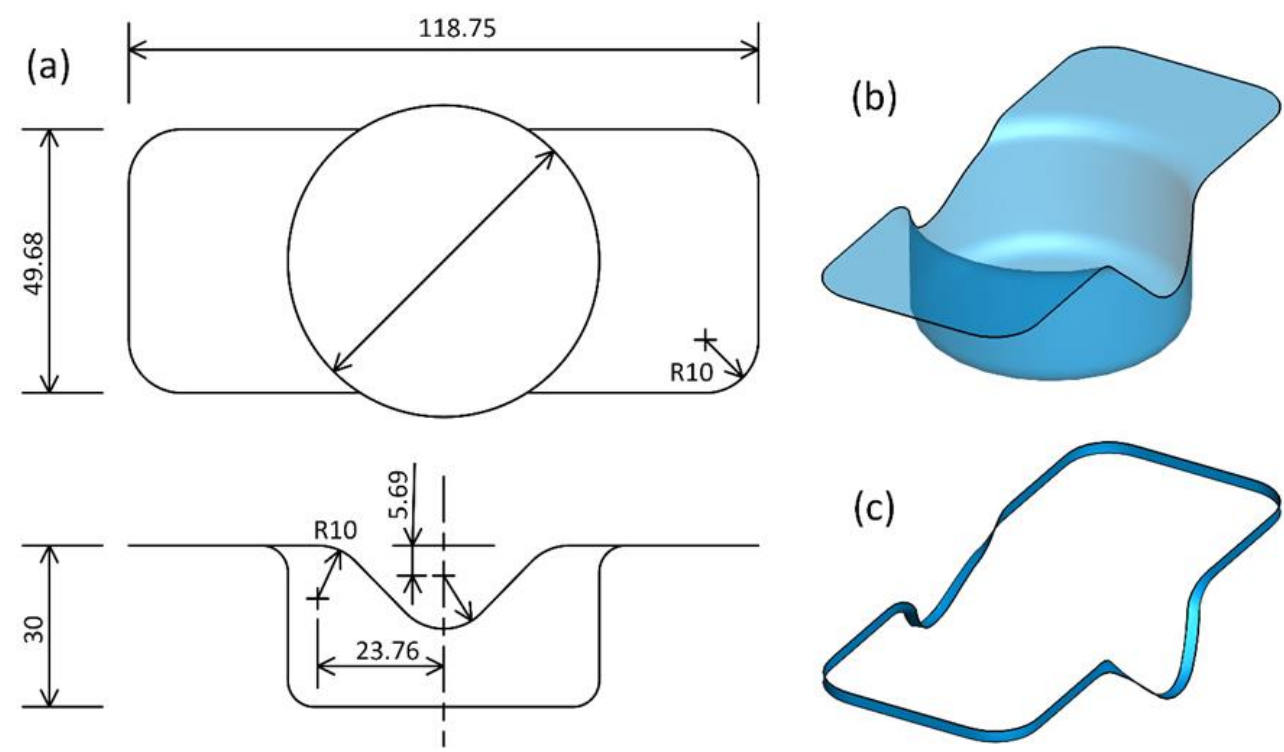

Figure 14: The geometric details of the target shape for example 3; (a) the front and top view of the target final product; (b) the 3D view of the target product; (c) The target contour.

The problem is solved using the proposed optimization procedure and the first three steps are given in Figure 15. In Figure 15(a), the initial guess for the blank is shown, which is selected as a rectangular shape with round corners. The deformed plate is also shown in this figure. It is visible that the final product is far from the target shape for the initial guess. The material flow paths of the boundary points are shown in Figure15 (b). As observed, the material flow paths cross the target ribbon in some points and there are other points that the flow path fails to reach the target. The shape errors are calculated for all boundary points and the initial blank is modified. Figure 15(c) shows the modified initial blank, in which the first iteration is started. The deformed plate and the material flow path of the boundary points are illustrated in Figs. 15(c) and (d), respectively. As shown, the deformed plate is now closer to the target ribbon, although it fails to match the target completely. The second and third iterations are demonstrated in Figs. 15(e) to 15(h). The final contours converge to the target shape.

Finally, the optimum initial blank is obtained after 20 iterations, as shown in Figure 16. Figure 17 displays the maximum shape error for different iterations. The maximum shape error for the initial guess is more than $13 \mathrm{~mm}$, which decreases rapidly as the optimization steps proceeds. The maximum shape errors reach less than $1 \mathrm{~mm}$ at the 12th iteration and are about $0.4 \mathrm{~mm}$ for 20 iterations. 

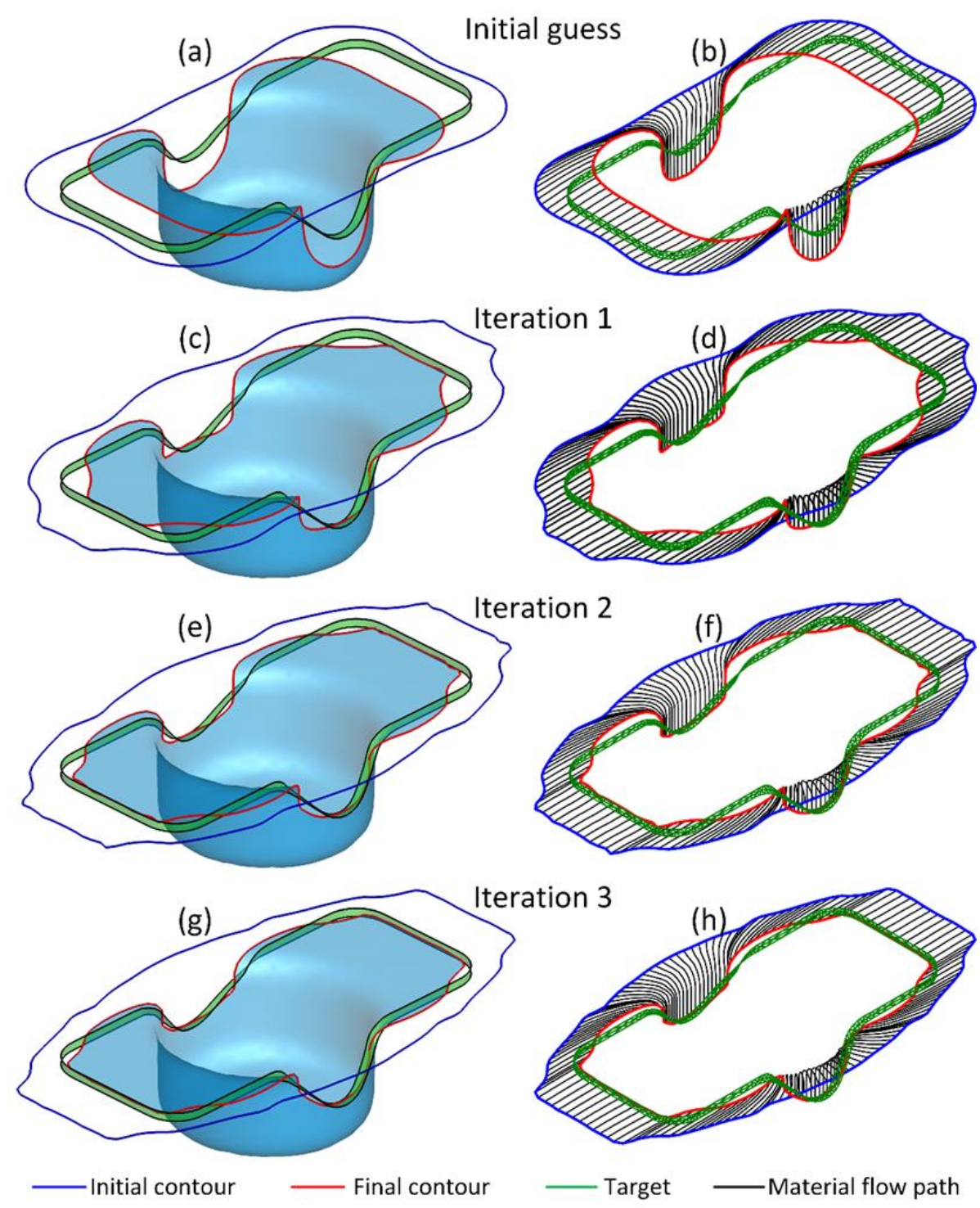

Figure 15: Results of example 3; (a) the initial guess for the blank; (b) The material flow paths of the boundary points; (c) The modified initial blank and the deformed plate; (d) material flow path of the boundary points; (e) to (h) The second and third iterations.

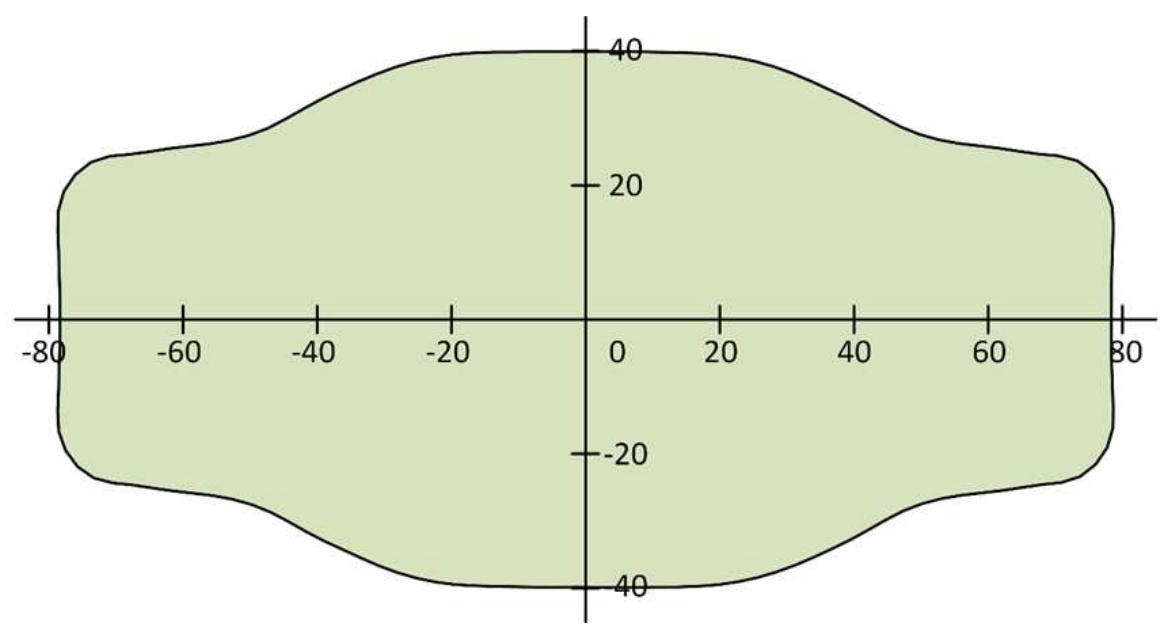

Figure 16: The optimum initial blank for example 3. 


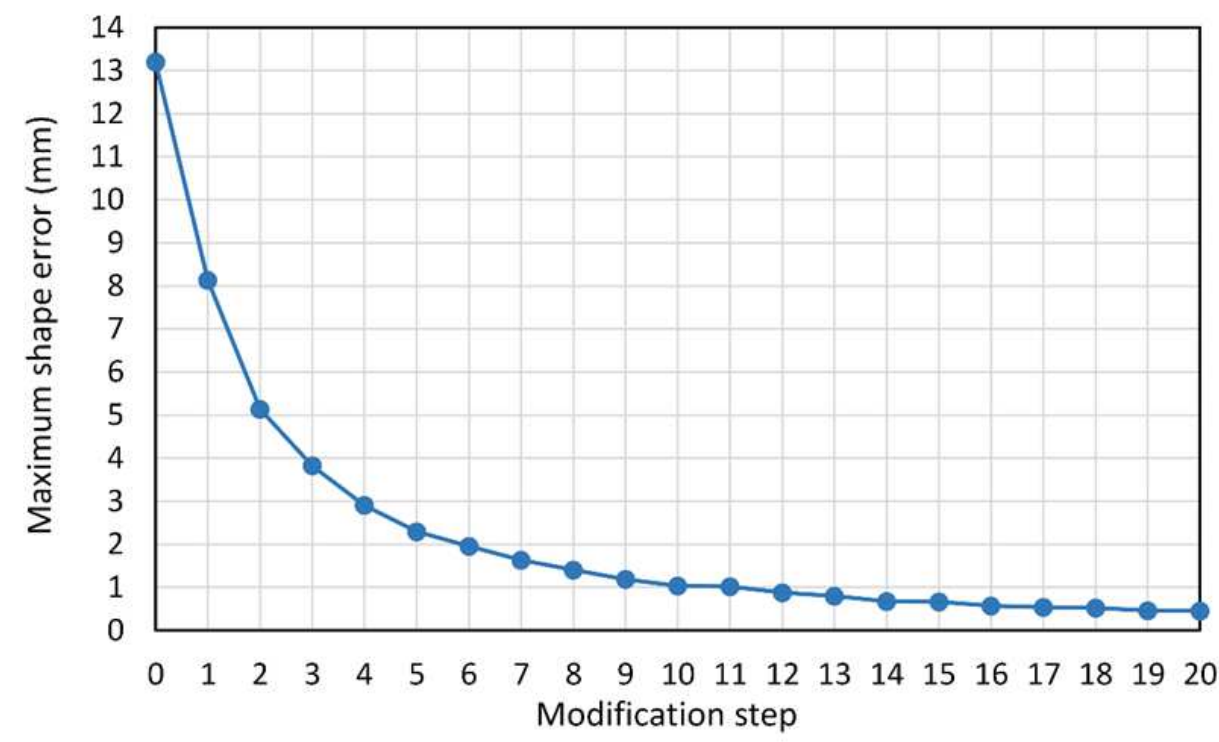

Figure 17: The maximum shape error for different iterations for example 3.

\subsection{Example 4: Unsymmetrical flange}

This example is another problem with a 3D target contour. The shape of the target product in the present example is different relative to the previous example and the flanges are not symmetric. The geometry of the punch and die, as well as other parameters, are similar to example 3. The geometric details of the target shape are illustrated in Figure 18. Specifically, Figure 18(a) shows the front and top view of the target final product while Figure 18(b) presents the $3 \mathrm{D}$ view of the target product. The target ribbon for the present example is also shown in Figure 18(c).

(a)
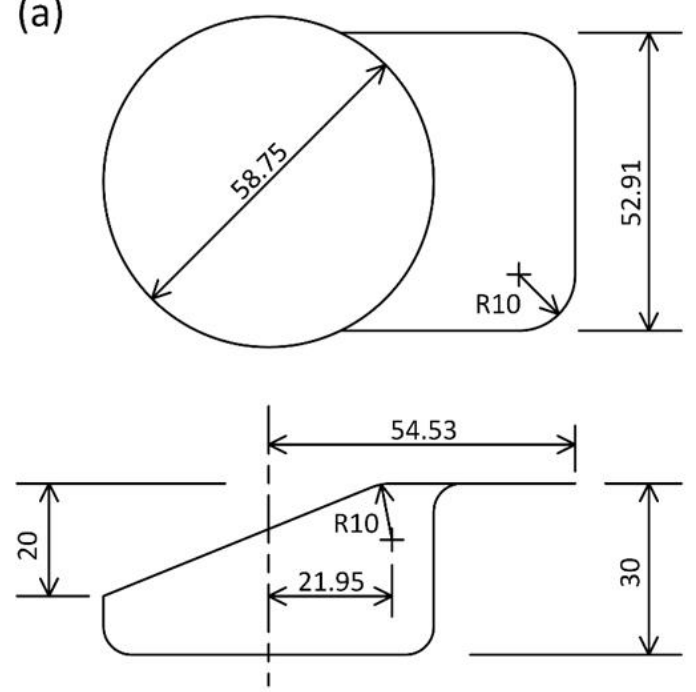
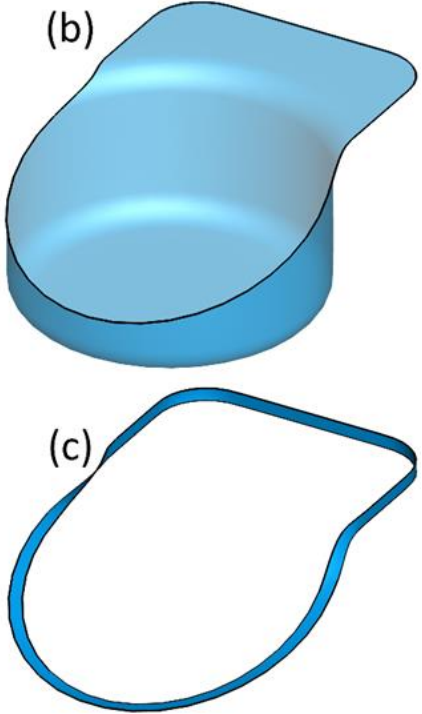

Figure 18: The geometric details of the target shape for example 4; (a) The front and top view of the target final product; (b) The 3D view of the target product; (c) The target ribbon. 
The problem is solved using the proposed optimization procedure, the first three steps of which are given in Figure 19. Figure 19(a) depicts the initial guess for the blank. The initial blank is selected as a rectangular shape with round corners. The deformed plate is also shown in this figure. It is observed that the final product is far from the target shape for the initial guess. The material flow paths of the boundary points are plotted in Fig .19(b). The material flow paths cross the target ribbon in some points while it fails to reach the target in some other points. The shape errors are calculated for all boundary points and the initial blank is modified, which is shown in Figure 19(c) and the first iteration is started. The deformed plate and the material flow path of the boundary points are given in Figs. 19(c) and (d), respectively. As observed, the deformed plate is now closer to the target ribbon, although it fails to match the target completely. The second and third iterations are given in Figs. 19(e) to 19(h), in which the final contours converge to the target shape.
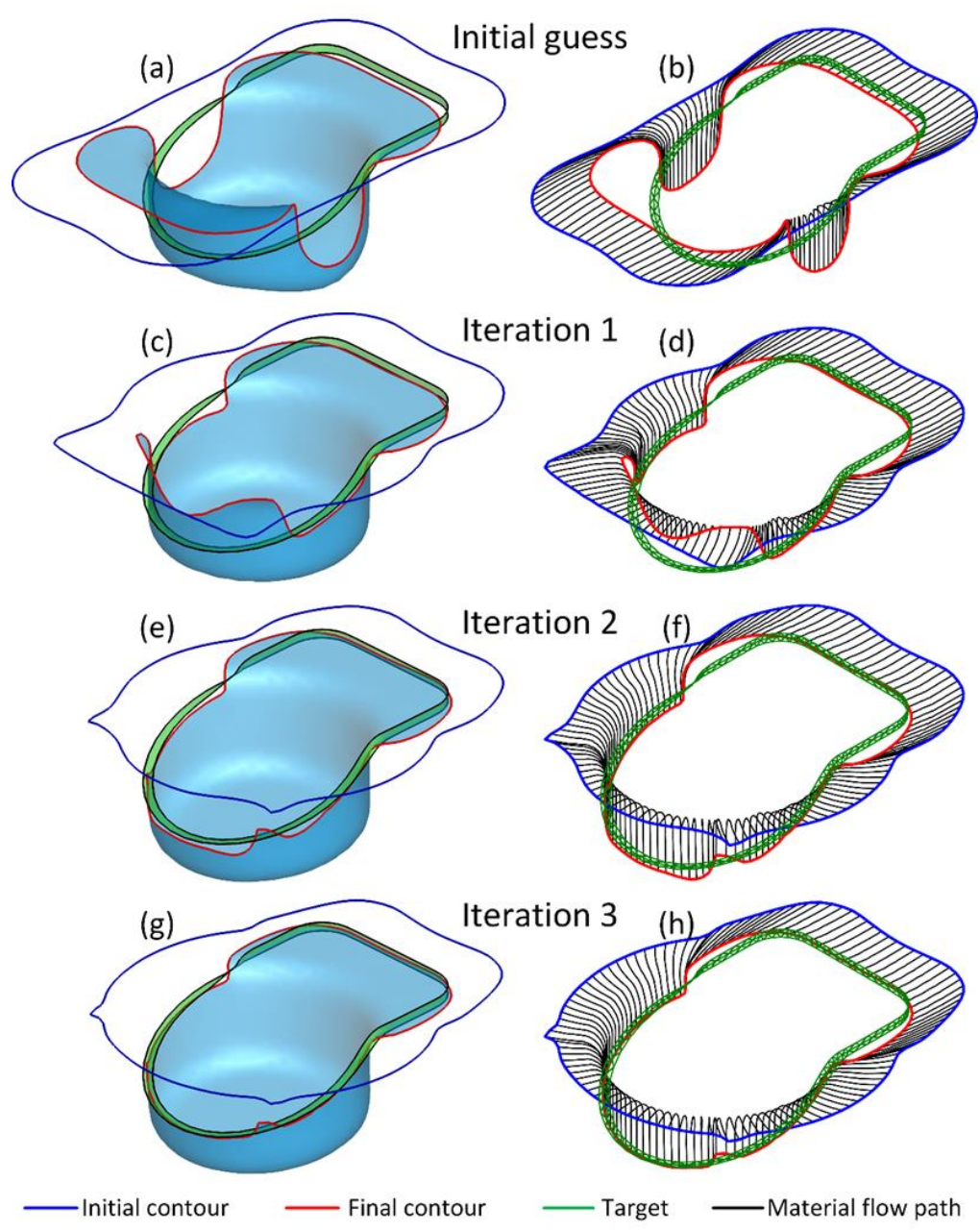

Figure 19: First three steps for example 4; (a) The initial guess for the blank and the deformed plate; (b) The material flow paths of the boundary points; (c) The modified initial blank and the deformed plate; (d) The material flow path of the boundary points; (e) to (h) The second and third iterations. 
Finally, the optimum initial blank is obtained after 20 iterations, as shown in Figure 20.

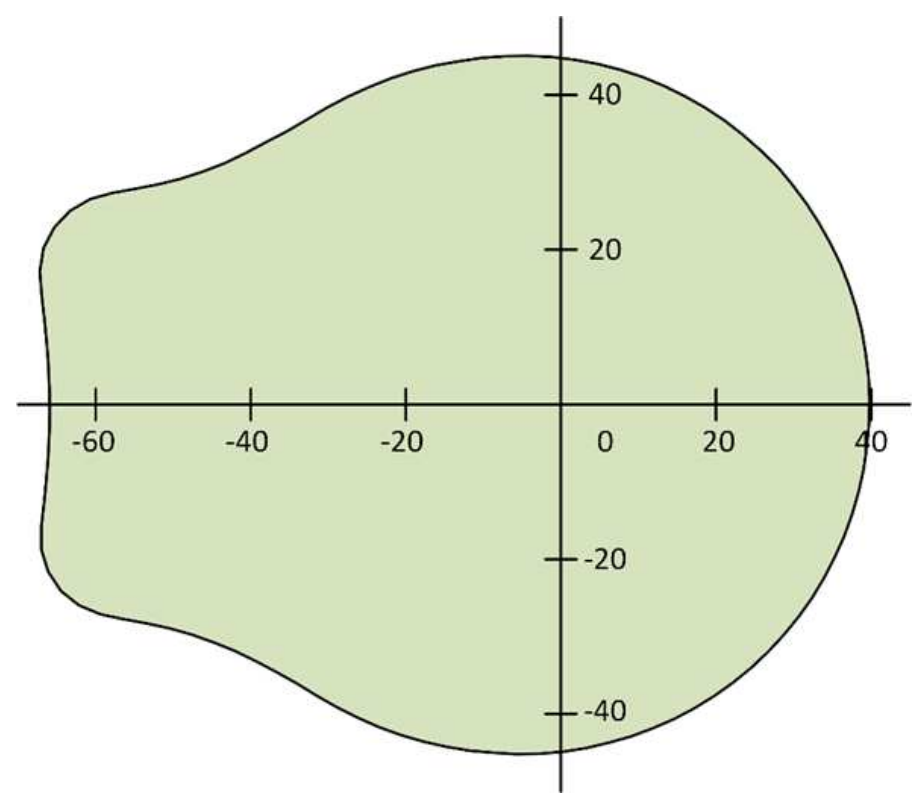

Figure 20: The optimum initial blank for example 4.

Figure 21 provides the maximum shape error for different iterations. As concluded, the maximum shape error for the initial guess is more than $26 \mathrm{~mm}$ but it decreased rapidly as the optimization steps proceeds. The maximum shape errors reach less than $1 \mathrm{~mm}$ at the 12 th iteration and are about $0.4 \mathrm{~mm}$ for 20 iterations.

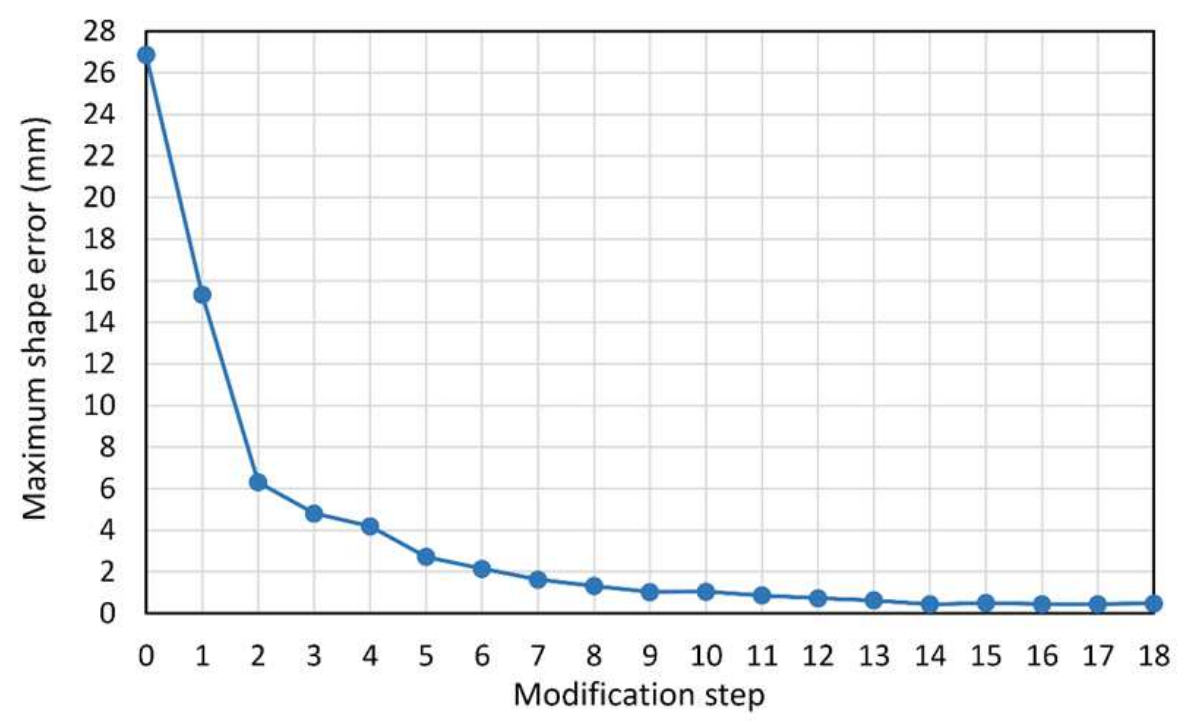

Figure 21: The maximum shape error for different iterations for example 4 


\subsection{Example 5: blank with internal boundary}

The last example addresses the sheet metal forming of a perforated plate. The blank has an internal boundary and the optimization problem should obtain both internal and external boundaries in such a way that the final product matches the internal and external targets. The geometry of the punch and the die and other parameters are similar to example 3 . The geometric details of the target shape and internal and external targets are given in Figure 22. Specifically, Figure 22(a) shows the front and top view of the target final product while Figure 22(b) illustrates the 3D view of the target product. Ultimately, Figure 22(c) depicts the target ribbons for the internal and external targets.
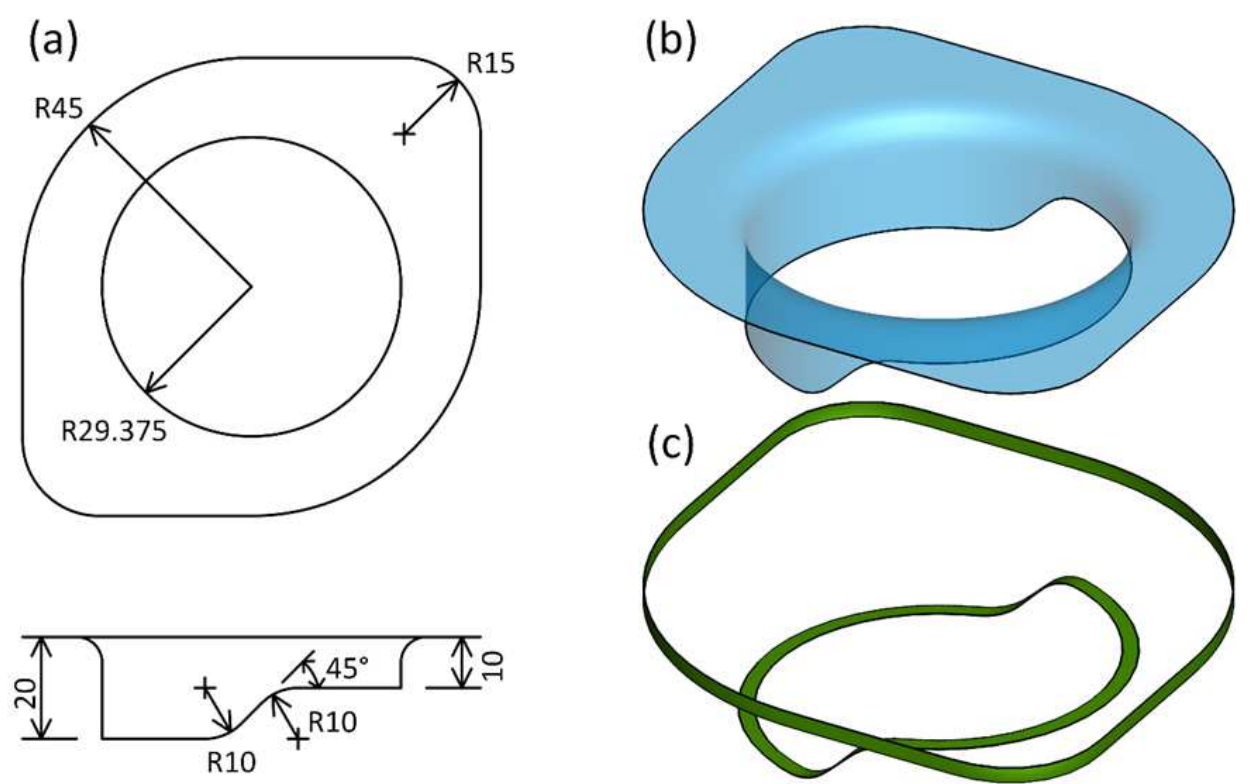

Figure 22: The geometric details of the target shape and internal and external targets for example 5;

(a) The front and top view of the target final product; (b) The 3D view of the target product; (c) The target ribbons for the internal and external targets.

The problem is solved using the proposed optimization procedure. Figure 23 provides the three iterations 2, 4, and 6. Figure 23(a) shows the initial guess for the blank, which is selected as an annulus with circular internal and external boundaries. The deformed plate is also shown in this figure. It is observed that the final product is far from the target shape for the initial guess. The material flow paths of the boundary points are illustrated in Figure 23(b). The shape errors are calculated for all boundary points on both internal and external boundaries and the initial blank is modified, which is shown in Figure 23(c) and is started in the first iteration. The deformed plate and the material flow path of the boundary points are depicted in Figs. 23(c) and (d), respectively. Accordingly, the deformed plate is now closer to the target ribbon 
although it fails to match the target completely. The fourth and sixth iterations are provided in Figs. 23(e) to 23(h), in which the final contours converge to the target shape.
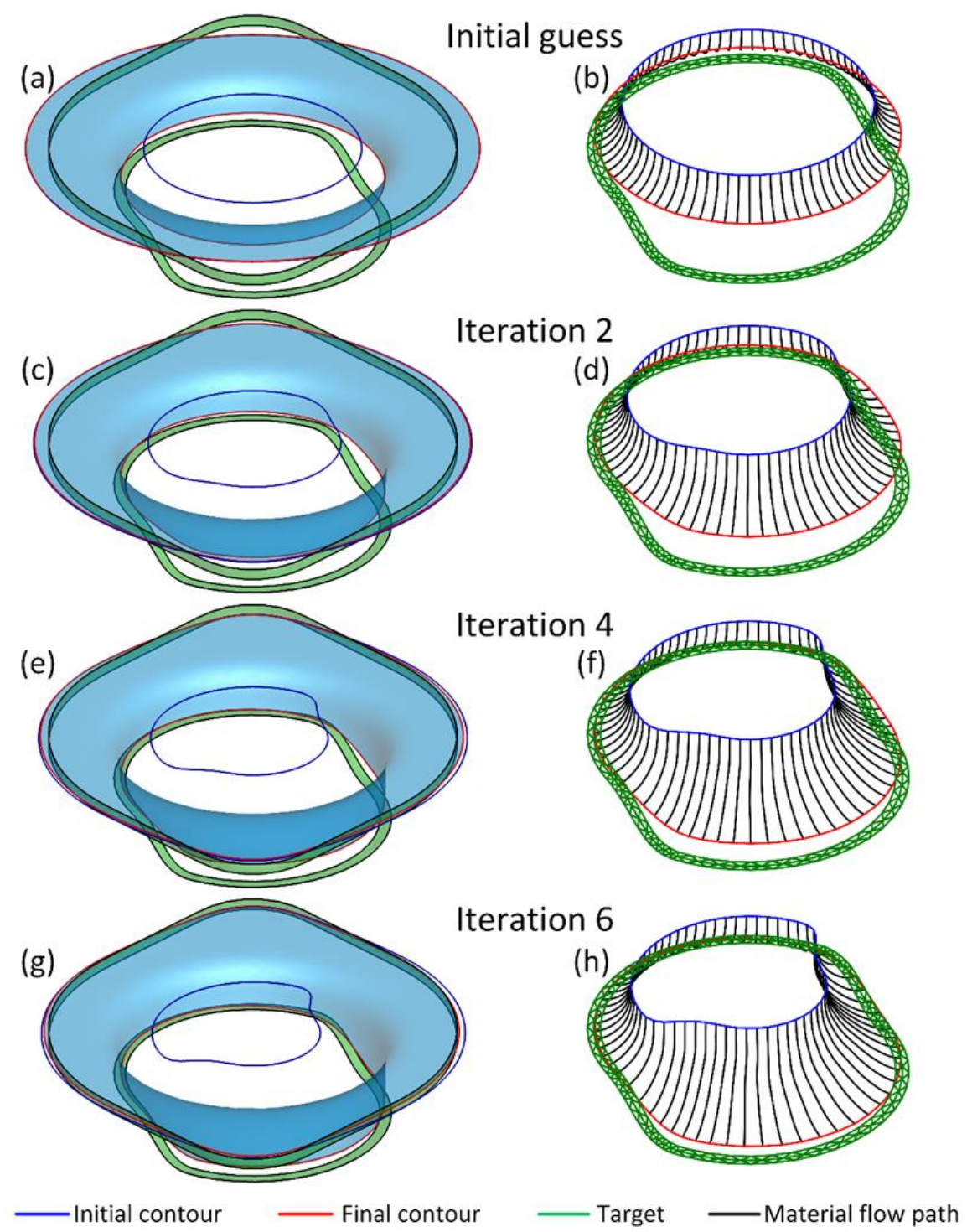

Figure 23: Results of example 5 (three iterations 2, 4 and 6); (a) The initial guess for the blank and the deformed plate; (b) The material flow paths of the boundary points; (c) The modified initial blank and the deformed plate; (d) The material flow path of the boundary points; (e) to (h) The fourth and sixth iterations.

Finally, the optimum initial blank is obtained after 20 iterations, as shown in Figure 24. The maximum shape error for different iterations is given in Figure 25. This figure shows that the maximum shape error for the initial guess is $13 \mathrm{~mm}$ for internal boundary and more than $7 \mathrm{~mm}$ for external boundary although it decreased rapidly as the optimization steps proceeds. Based on the results, the maximum shape errors reach less than $1 \mathrm{~mm}$ at the 12 th iteration and are about $0.4 \mathrm{~mm}$ for 20 iterations. 


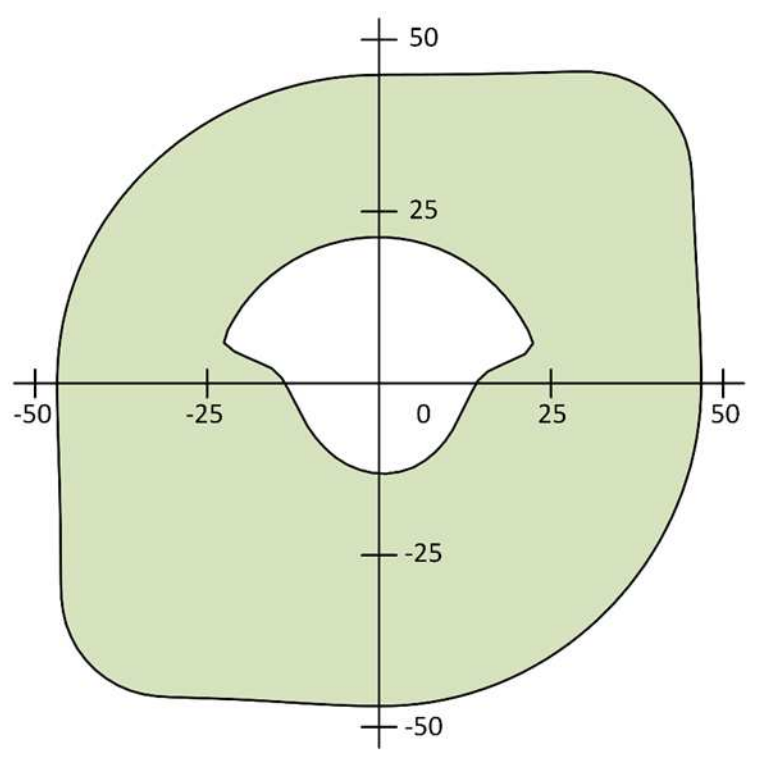

Figure 24: The optimum initial blank for example 5.

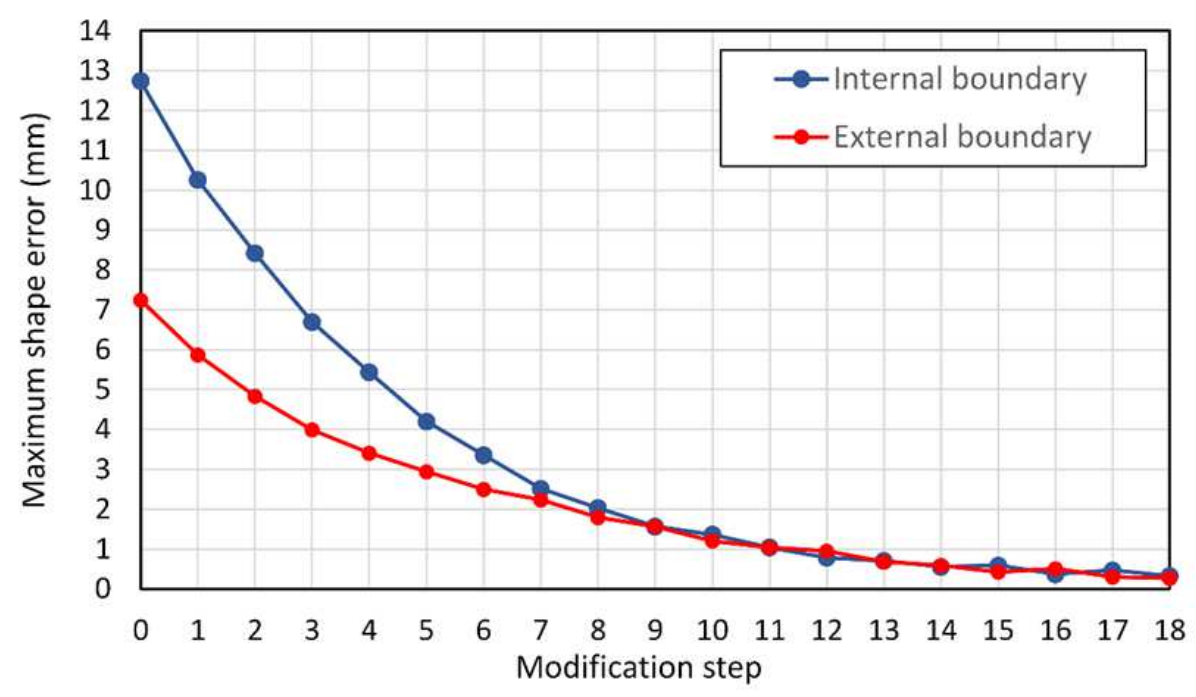

Figure 25: The maximum shape error for different iterations for example 5.

\section{Conclusion}

The present study sought to propose a new blank optimization algorithm for deep drawing, aiming to develop an algorithm that is robust against the initial guess and can find the solution to the blank optimization problem for the deep drawing process, by starting from a far-off initial guess. In the proposed algorithm, the shape of the initial blank was outlined by several key points, and then, the iterative process started from an initial guess to get the optimum shape. The process continued until realizing the stopping criterion. The efficiency of the proposed method was evaluated by solving five examples. Further, by considering a cavity inside the blank, the external and internal boundaries were corrected simultaneously as a problem using 
the proposed algorithm, resulting in proving the effectiveness of the algorithm for correcting internal boundaries. This approach can be useful in the production of parts that feature cavities as the blank can be prepared with a cavity of a particular geometry that can develop the desired shape by deformation during deep drawing. This method is especially useful in cases with limitations in creating holes after the drawing process. The results showed that the proposed algorithm is sufficiently robust against the initial guesses for the blank, which is an advantage of the present algorithm compared to other algorithms. Finally, the present study reveals that the proposed algorithm can be effectively used to solve blank optimization problems for the deep drawing process.

Funding: The authors did not receive support from any organization for the submitted work.

Conflict of Interest: The authors declare that they have no conflict of interest.

Ethical approval: This article does not contain any studies with human participants or animals performed by any of the authors.

\section{References}

[1] M.H. Parsa, P.H. Matin, M.M. Mashhadi, Improvement of initial blank shape for intricate products using slip line field, J. Mater. Process. Technol. 145 (2004) 21-26. https://doi.org/10.1016/S0924-0136(03)00858-6.

[2] T. Kuwabara, W. Si, PC-based blank design system for deep-drawing irregularly shaped prismatic shells with arbitrarily shape flange, J. Mater. Process. Technol. 63 (1997) 8994. https://doi.org/10.1016/S0924-0136(96)02605-2.

[3] X. Chen, R. Sowerby, Blank development and the prediction of earing in cup drawing, Int. J. Mech. Sci. 38 (1996) 509-516. https://doi.org/10.1016/0020-7403(95)00068-2.

[4] X. Chen, R. Sowerby, The development of ideal blank shapes by the method of plane stress characteristics, Int. J. Mech. Sci. 34 (1992) 159-166. https://doi.org/10.1016/0020-7403(92)90080-Z.

[5] J.H. Vogel, D. Lee, An analysis method for deep drawing process design, Int. J. Mech. Sci. 32 (1990) 891-907. https://doi.org/10.1016/0020-7403(90)90062-N.

[6] M. Karima, Blank Development and Tooling Design for Drawn Parts Using a Modified Slip Line Field Based Approach, J. Eng. Ind. 111 (1989) 345-350. https://doi.org/10.1115/1.3188770.

[7] H. Gloeckl, Computer aided design of blanks for deep drawn irregular shaped components, in: Proc. 11th NAMRC, SME, 1983. 
[8] R. Sowerby, N. Chandrasekharan, X. Chen, M. Rooks, P. Correa, The development of computer aids for sheet metal metal stampings, in: CAD/CAM FEM Met. 3rd Int. Conf. SAS, Pergamon Press, 1988: p. 187.

[9] V. V Hazek, Use of the slip line field method in deep drawing large irregular shaped components, in: Proc. 7th NAMRC, SME, 1979.

[10] N. Kim, S. Kobayashi, Blank design in rectangular cup drawing by an approximate method, Int. J. Mach. Tool Des. Res. 26 (1986) 125-135. https://doi.org/10.1016/00207357(86)90213-1.

[11] R. Sowerby, J.L. Duncan, E. Chu, The modelling of sheet metal stampings, Int. J. Mech. Sci. 28 (1986) 415-430. https://doi.org/10.1016/0020-7403(86)90062-7.

[12] G.N. BLOUNT, B. V FISCHER, Computerised blank shape prediction for sheet metal components having doubly-curved surfaces, Int. J. Prod. Res. 33 (1995) 993-1005. https://doi.org/10.1080/00207549508930190.

[13] A.M. Zaky, A.B. Nassr, M.G. El-Sebaie, Optimum blank shape of cylindrical cups in deep drawing of anisotropic sheet metals, J. Mater. Process. Technol. 76 (1998) 203211. https://doi.org/10.1016/S0924-0136(97)00349-X.

[14] K. Chung, O. Richmond, Ideal forming - II. Sheet forming with optimum deformation, Int. J. Mech. Sci. 34 (1992) 617-633. https://doi.org/10.1016/0020-7403(92)90059-P.

[15] H. Iseki, R. Sowerby, Determination of the Optimum Blank Shape When Deep Drawing Nonaxisymmetric Cups, Using a Finite-Element Method: Simplified Determination of Optimum Blank Shape and Limiting Drawing Ratio by Inverse Finite-Element Procedure, JSME Int. Journal. Ser. A, Mech. Mater. Eng. 38 (1995) 473-479. https://doi.org/10.1299/jsmea1993.38.4_473.

[16] R. Azizi, Different implementations of inverse finite element method in sheet metal forming, Mater. $\quad$ Des. $30 \quad$ (2009) 2975-2980. https://doi.org/10.1016/j.matdes.2008.12.022.

[17] O. Barlet, J.L. Batoz, Y.Q. Guo, F. Mercier, H. Naceur, C. Knopf-Lenoir, Optimum design of blank contours using the inverse approach and a mathematical programming technique, in: Numisheet, 1996: pp. 178-185.

[18] C.H. Lee, H. Huh, Blank design and strain prediction of automobile stamping parts by an inverse finite element approach, J. Mater. Process. Technol. 63 (1997) 645-650. https://doi.org/10.1016/S0924-0136(96)02700-8.

[19] Y.Q. Guo, J.L. Batoz, H. Naceur, S. Bouabdallah, F. Mercier, O. Barlet, Recent developments on the analysis and optimum design of sheet metal forming parts using a 
simplified inverse approach, Comput. Struct. $78 \quad$ (2000) 133-148. https://doi.org/10.1016/S0045-7949(00)00095-X.

[20] T.W. Ku, H.J. Lim, H.H. Choi, S.M. Hwang, B.S. Kang, Implementation of backward tracing scheme of the FEM to blank design in sheet metal forming, J. Mater. Process. Technol. 111 (2001) 90-97. https://doi.org/10.1016/S0924-0136(01)00518-0.

[21] H. Naceur, Y.Q. Guo, J.L. Batoz, Blank optimization in sheet metal forming using an evolutionary algorithm, J. Mater. Process. Technol. 151 (2004) 183-191. https://doi.org/10.1016/j.jmatprotec.2004.04.036.

[22] Z.-Y. Cai, M.-Z. Li, H.-M. Zhang, A simplified algorithm for planar development of 3D surface and its application in the blank design of sheet metal forming, Finite Elem. Anal. Des. 43 (2007) 301-310. https://doi.org/10.1016/j.finel.2006.10.005.

[23] M.H. Parsa, P. Pournia, Optimization of initial blank shape predicted based on inverse finite element method, Finite Elem. Anal. Des. 43 (2007) 218-233. https://doi.org/10.1016/j.finel.2006.09.005.

[24] R. Azizi, A. Assempour, Applications of linear inverse finite element method in prediction of the optimum blank in sheet metal forming, Mater. Des. 29 (2008) 19651972. https://doi.org/10.1016/j.matdes.2008.04.015.

[25] C.H. Toh, S. Kobayashi, Deformation analysis and blank design in square cup drawing, Int. J. Mach. Tool Des. Res. 25 (1985) 15-32. https://doi.org/10.1016/00207357(85)90054-X.

[26] K. Chung, F. Barlat, J.C. Brem, D.J. Lege, O. Richmond, Blank shape design for a planar anisotropic sheet based on ideal forming design theory and FEM analysis, Int. J. Mech. Sci. 39 (1997) 105-120. https://doi.org/10.1016/0020-7403(96)00007-0.

[27] A. Fazli, B. Arezoo, A comparison of numerical iteration based algorithms in blank optimization, Finite Elem. Anal. Des. 50 (2012) 207-216. https://doi.org/10.1016/j.finel.2011.09.011.

[28] S.H. Park, J.W. Yoon, D.Y. Yang, Y.H. Kim, Optimum blank design in sheet metal forming by the deformation path iteration method, Int. J. Mech. Sci. 41 (1999) 12171232. https://doi.org/10.1016/S0020-7403(98)00084-8.

[29] V. Pegada, Y. Chun, S. Santhanam, An algorithm for determining the optimal blank shape for the deep drawing of aluminum cups, J. Mater. Process. Technol. 125-126 (2002) 743-750. https://doi.org/10.1016/S0924-0136(02)00382-5.

[30] H. Shim, K. Son, Optimal blank shape design by the iterative sensitivity method, Proc. Inst. Mech. Eng. Part B J. Eng. Manuf. 216 (2002) 867-878. 
https://doi.org/10.1243/095440502320192996.

[31] K. Son, H. Shim, Optimal blank shape design using the initial velocity of boundary nodes, J. Mater. Process. Technol. 134 (2003) 92-98. https://doi.org/10.1016/S09240136(02)00927-5.

[32] A. Vafaeesefat, Optimum blank shape design in sheet metal forming by boundary projection method, Int. J. Mater. Form. 1 (2008) 189-192. https://doi.org/10.1007/s12289-008-0023-2.

[33] M. Azaouzi, H. Naceur, A. Delamézière, J.L. Batoz, S. Belouettar, An Heuristic Optimization Algorithm for the blank shape design of high precision metallic parts obtained by a particular stamping process, Finite Elem. Anal. Des. 44 (2008) 842-850. https://doi.org/10.1016/j.finel.2008.06.008.

[34] W. Hammami, R. Padmanabhan, M.C. Oliveira, H. BelHadjSalah, J.L. Alves, L.F. Menezes, A deformation based blank design method for formed parts, Int. J. Mech. Mater. Des. 5 (2009) 303. https://doi.org/10.1007/s10999-009-9103-9.

[35] R. Padmanabhan, M.C. Oliveira, A.J. Baptista, J.L. Alves, L.F. Menezes, Numerical study on the influence of initial anisotropy on optimal blank shape, Finite Elem. Anal. Des. 45 (2009) 71-80. https://doi.org/10.1016/j.finel.2008.07.012. 
Figures
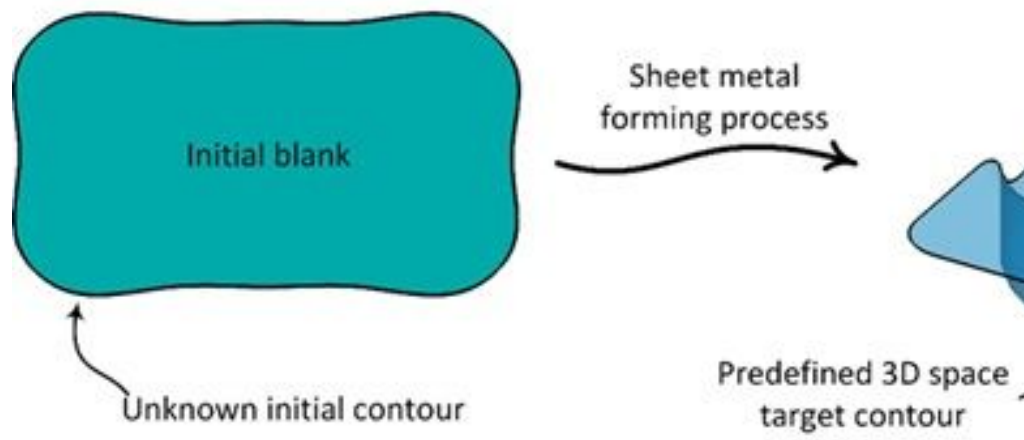

Figure 1

Initial blank shape optimization problem with 3D space target contour

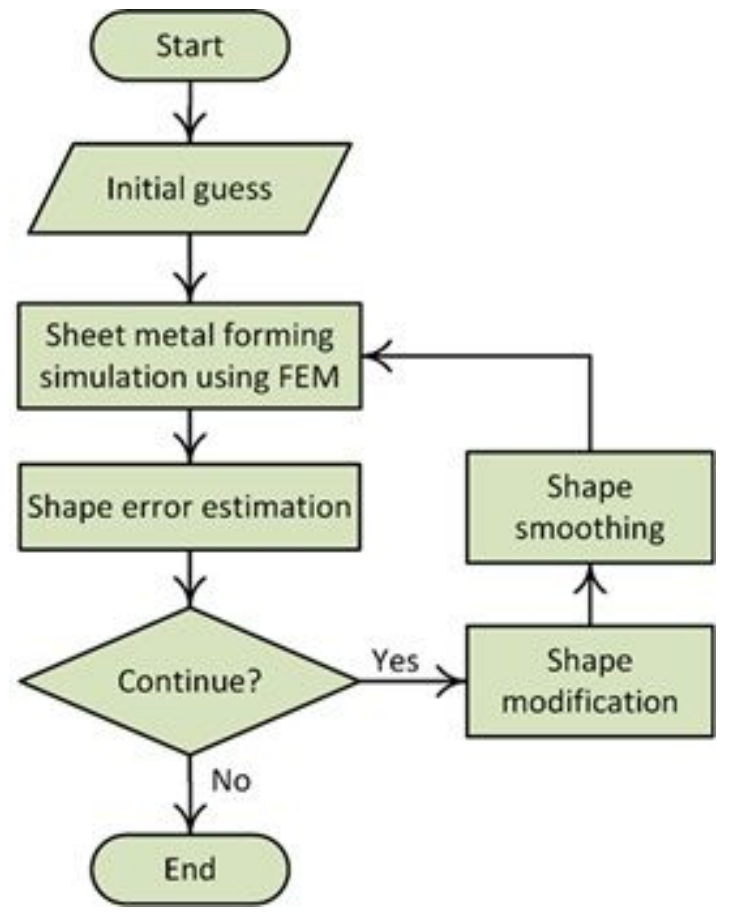

Figure 2

The flow chart of blank optimization by the iterative method based on numerical simulation
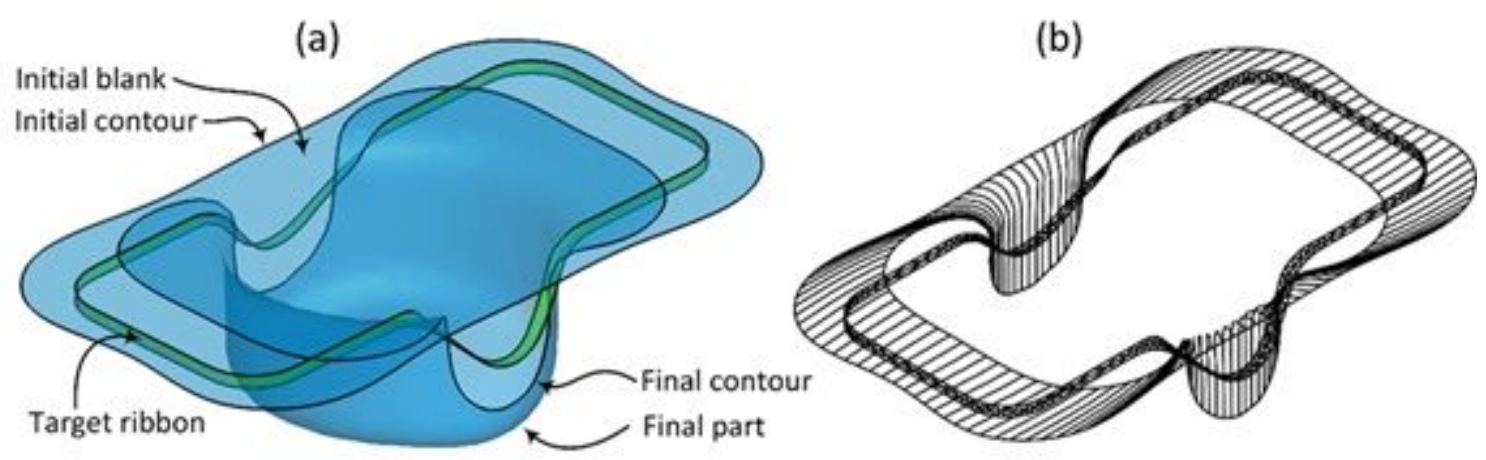
Figure 3

(a) A schematic representation of blank shape optimization problem with 3D space target; (b) The flow path of the material points from initial contour to final contour in the sheet metal forming process

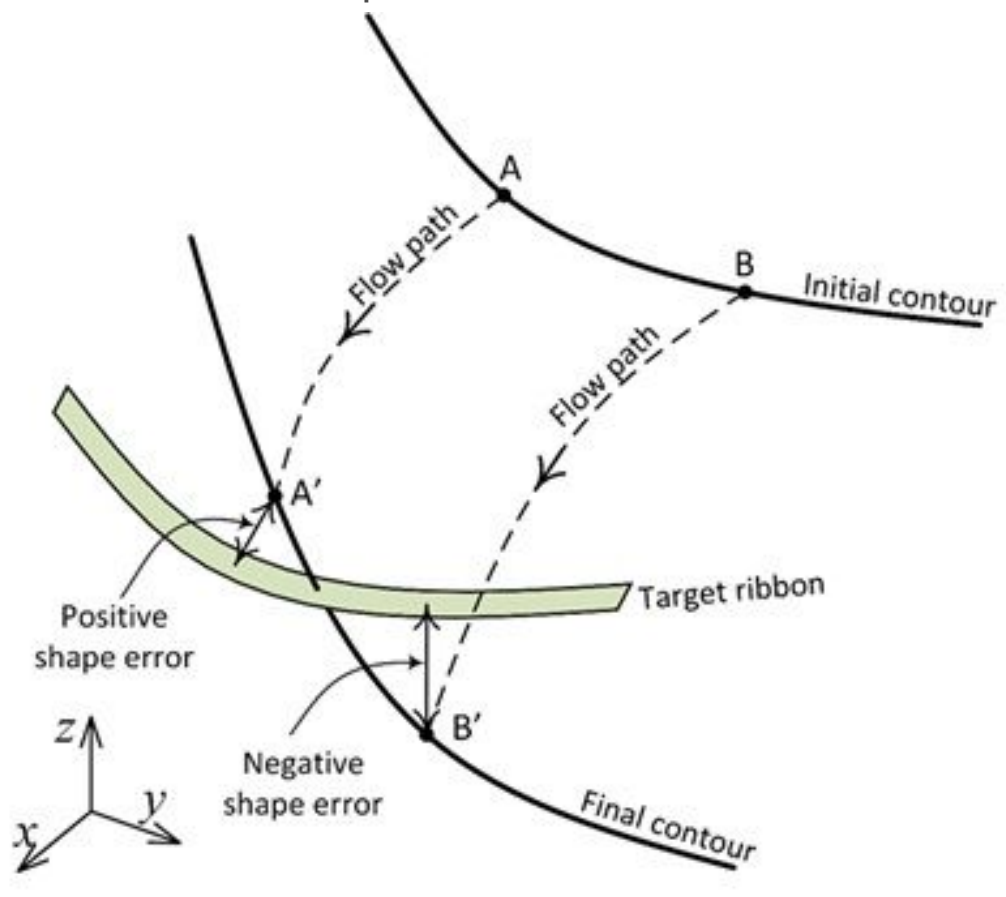

Figure 4

Definition of the shape error

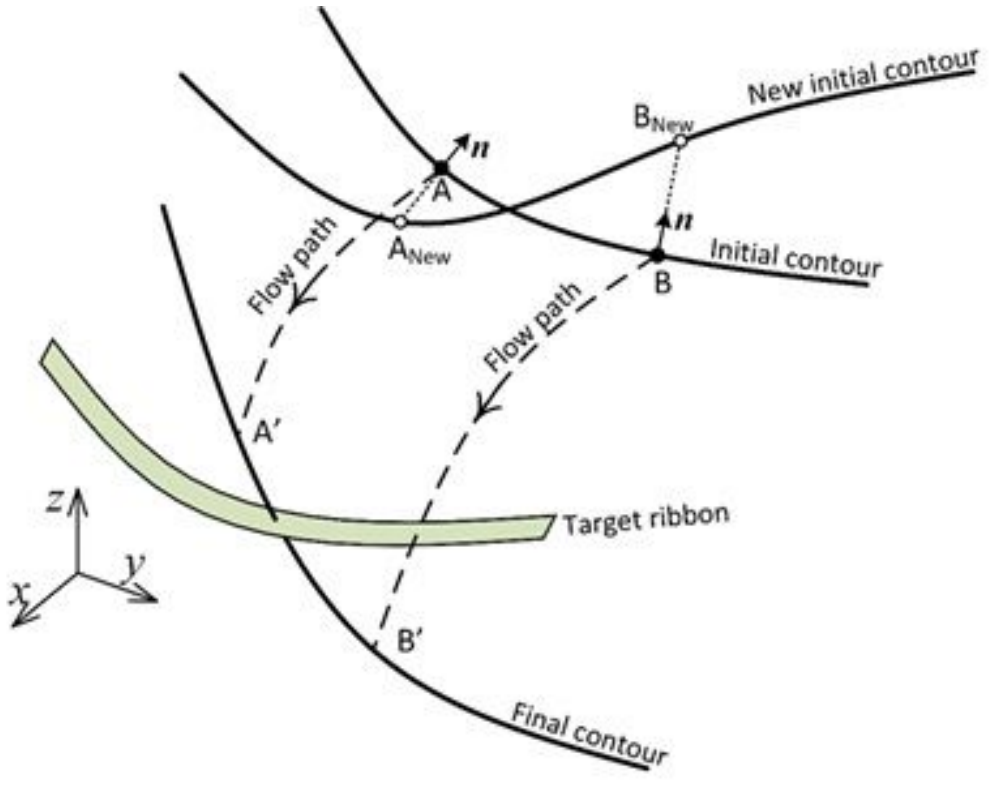

Figure 5

Modification of the initial contour 


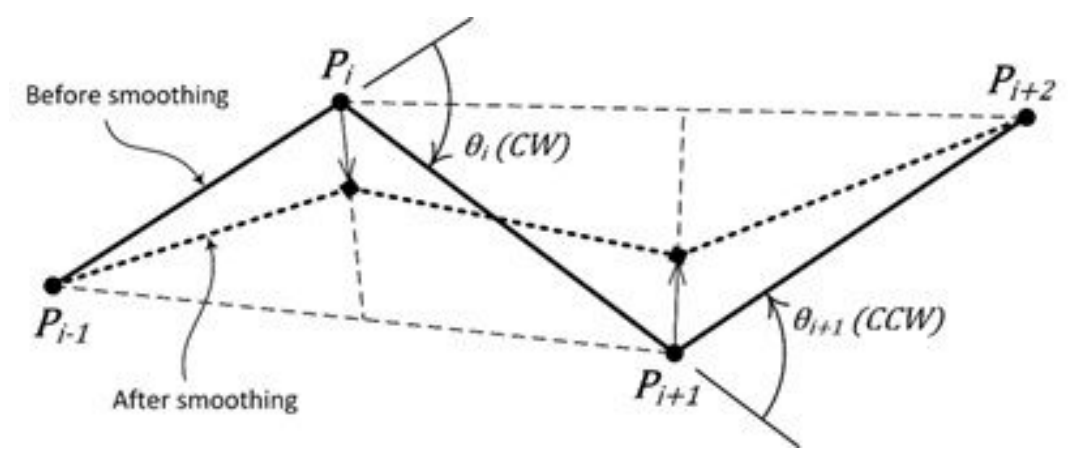

Figure 6

Smoothing the zigzag parts of the boundary
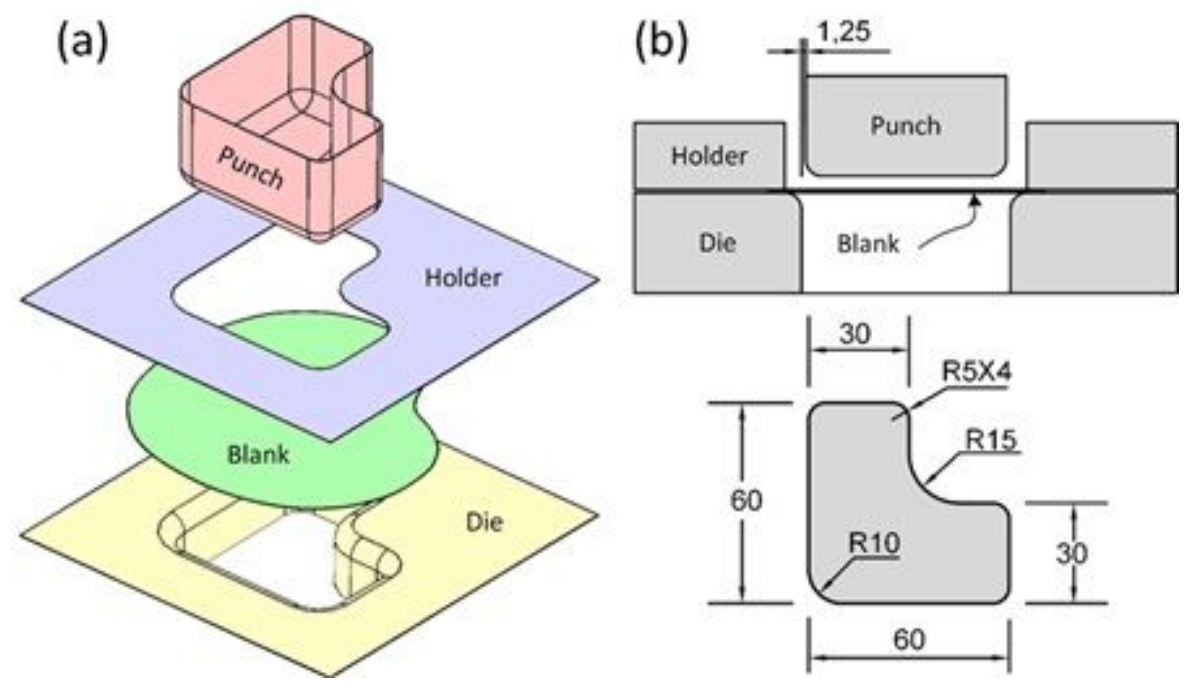

Figure 7

(a) Sheet metal forming tools assembly; (b) Dimensions of the tools for example 1 

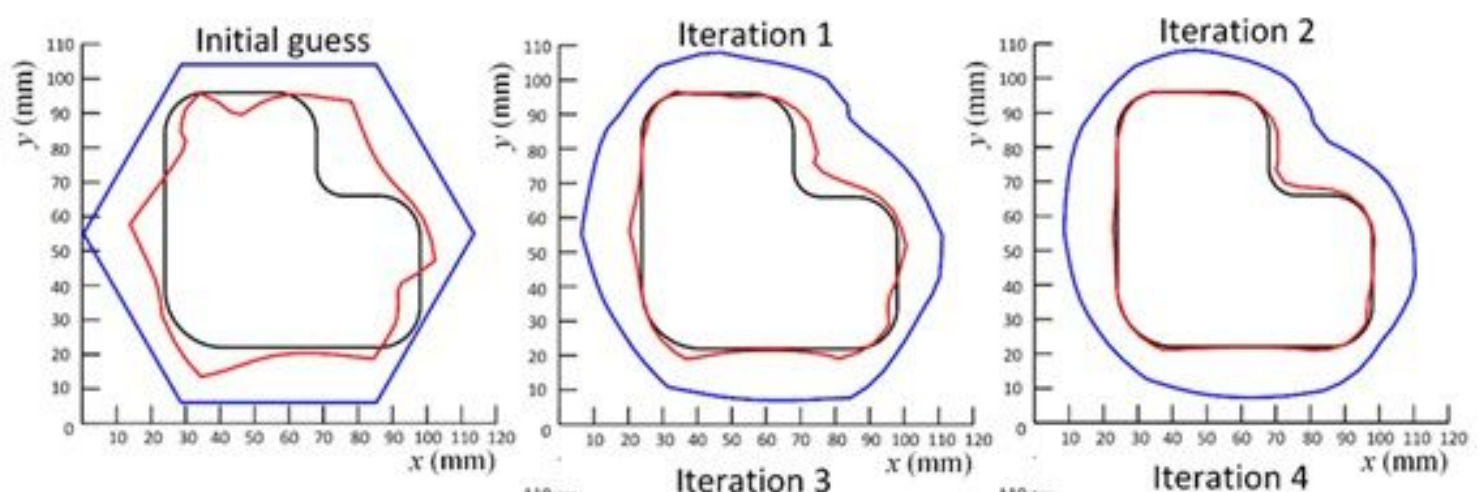

Initial contour

Final contour

Target contour
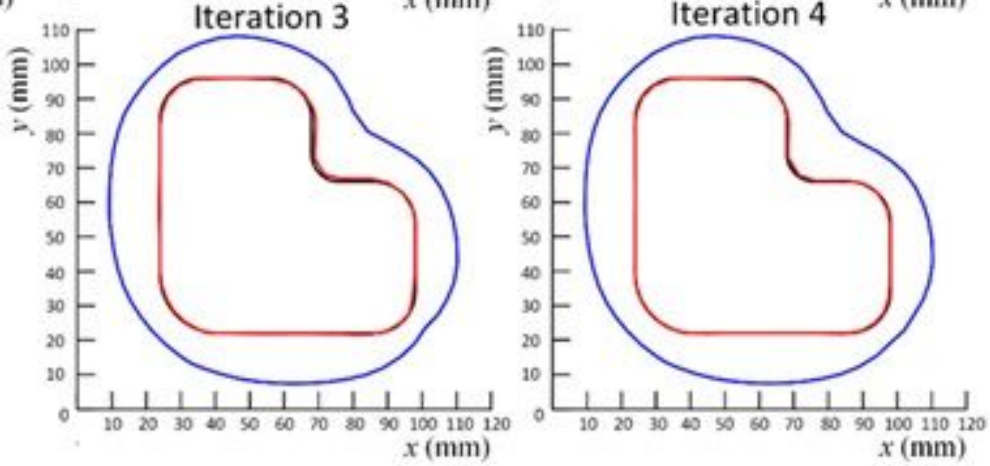

Figure 8

Initial and final contours for the first four iterations starting from a hexagonal initial guess for example 1.

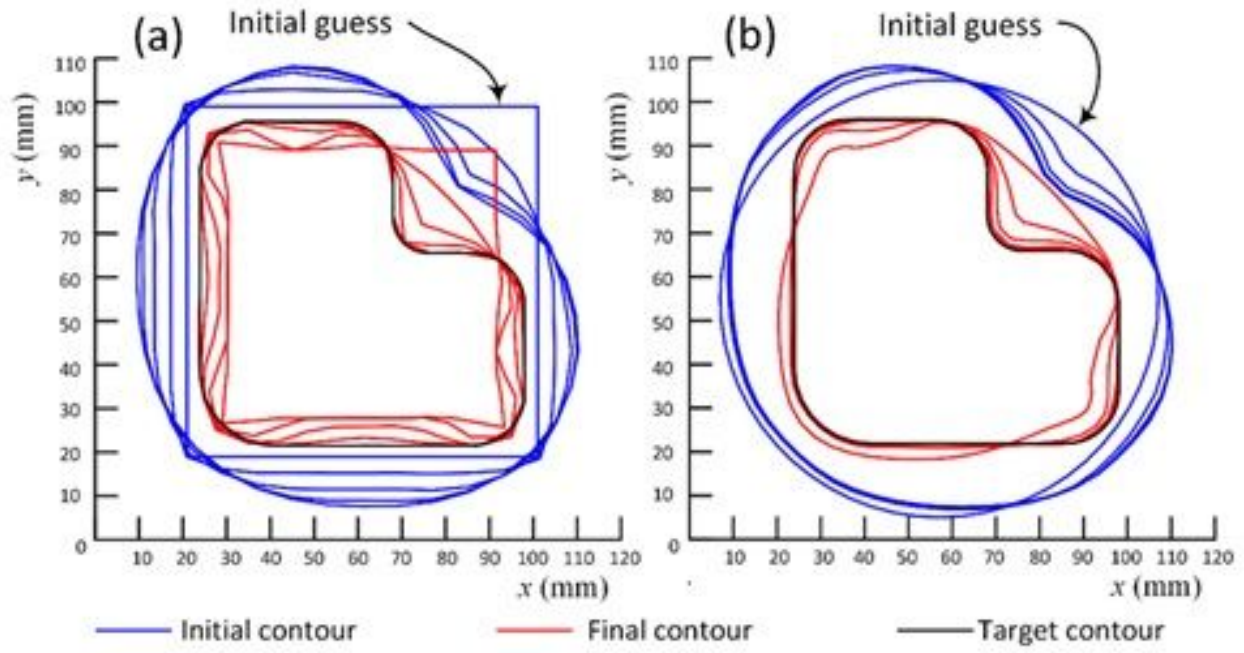

Figure 9

Initial and final contours for successive iteration of example 1; (a) starting from a squared initial guess; (b) starting from a circular initial guess. 


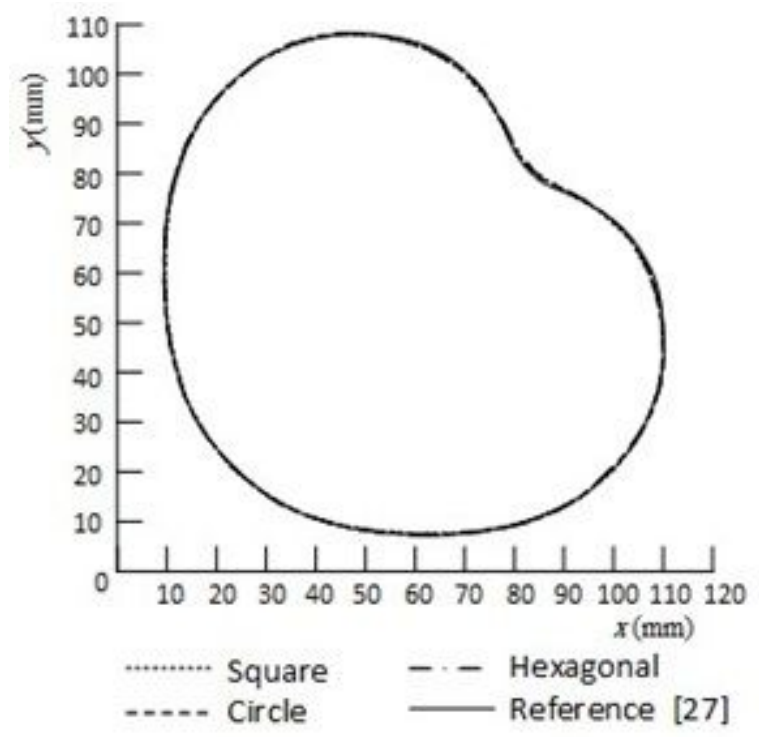

Figure 10

The optimum blanks obtained using three different initial guesses and comparing with the shape given in Reference [27].

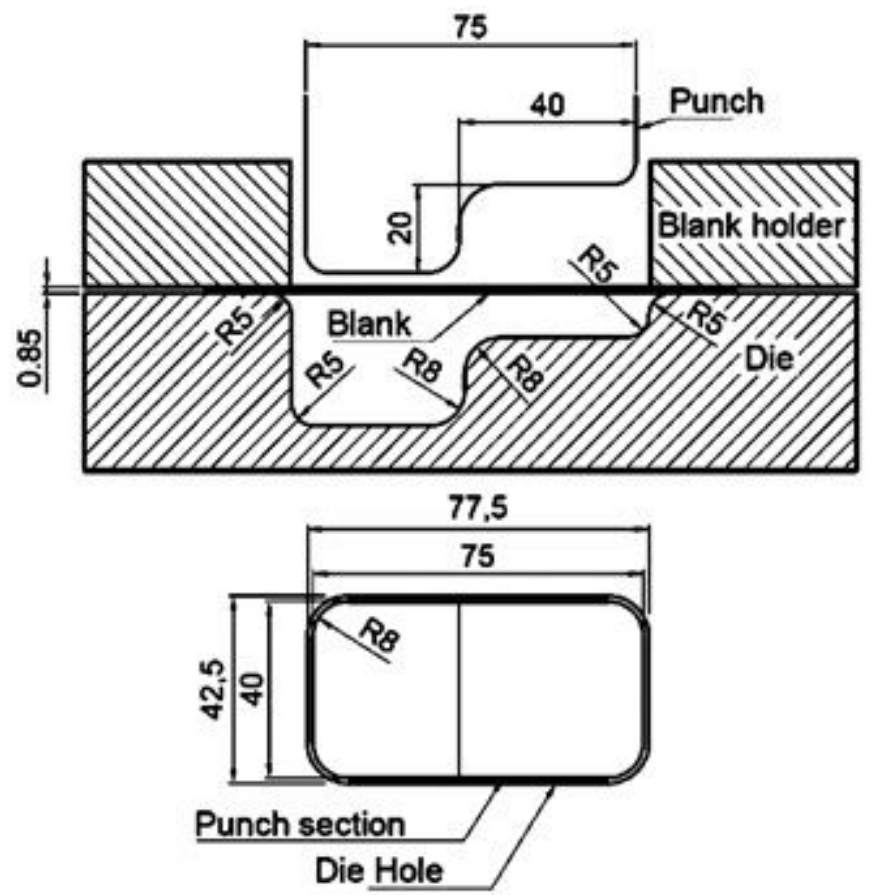

Figure 11

Dimensions of the tools for example 2. 
(a) Initial guesses
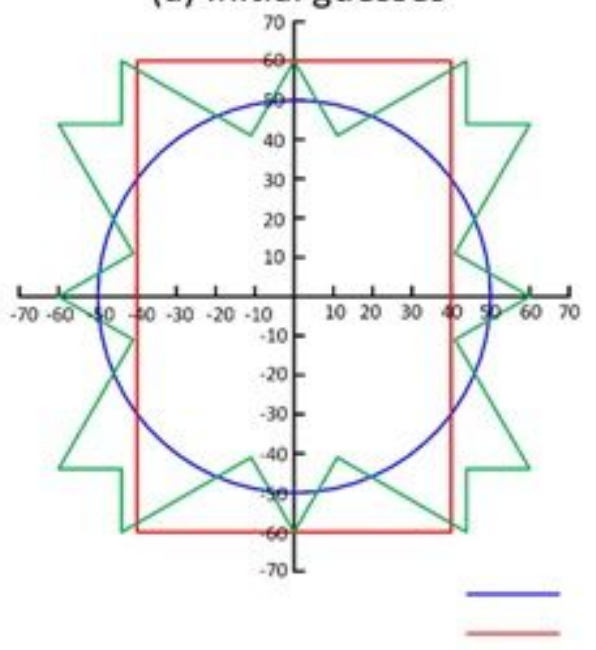

(b) Initial contoures

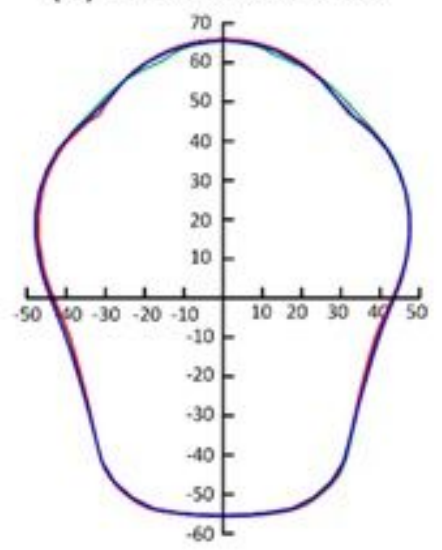

(c) final contoures

Circle

Rectangle

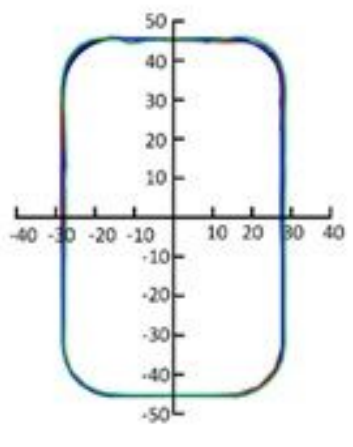

Zigzag

Target

\section{Figure 12}

Results of example 2; (a) Three different initial guesses for the initial blank; (b) The optimum initial blank obtained with three different initial guesses; (c) The final contours obtained from three different initial guesses.

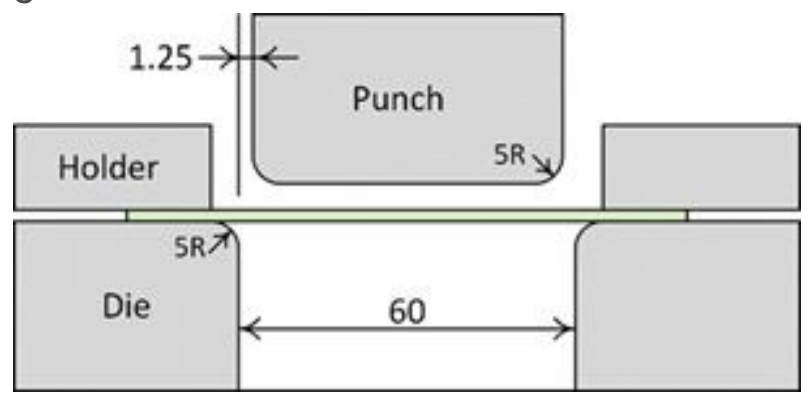

\section{Figure 13}

The geometric details of the tools for example 3.
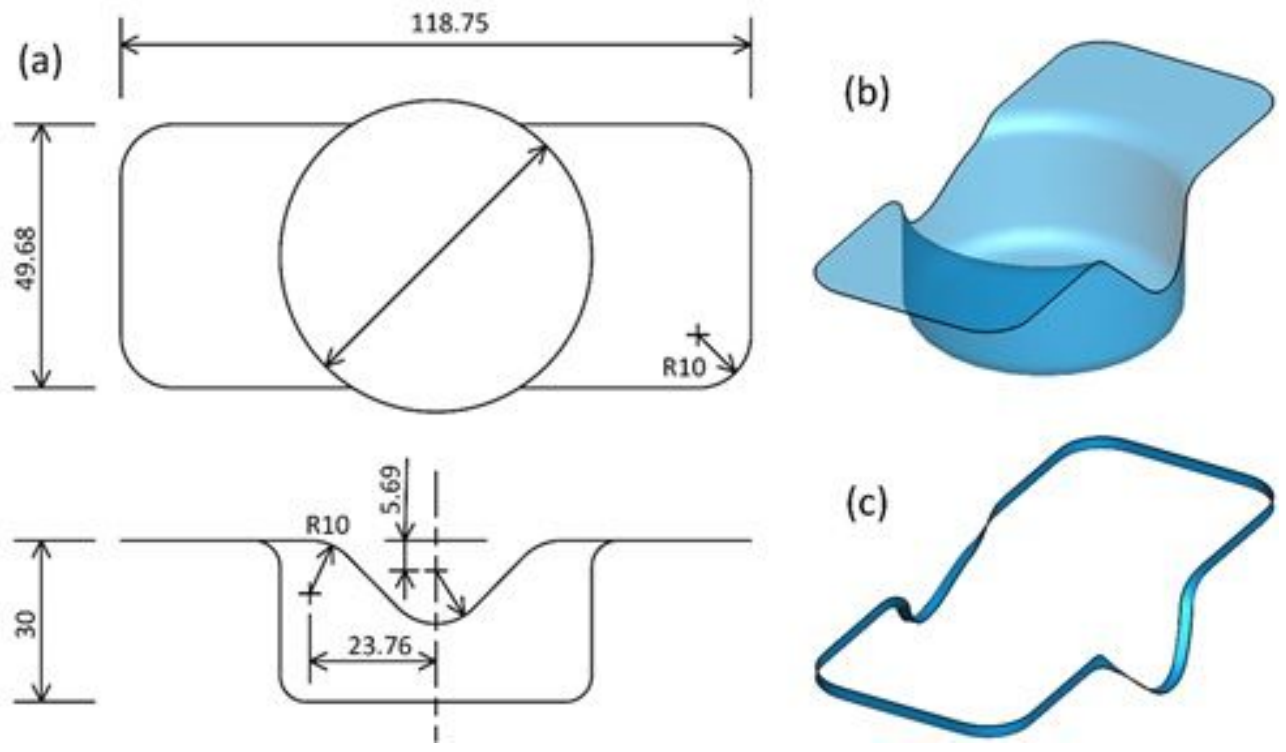


\section{Figure 14}

The geometric details of the target shape for example 3; (a) the front and top view of the target final product; (b) the 3D view of the target product; (c) The target contour.
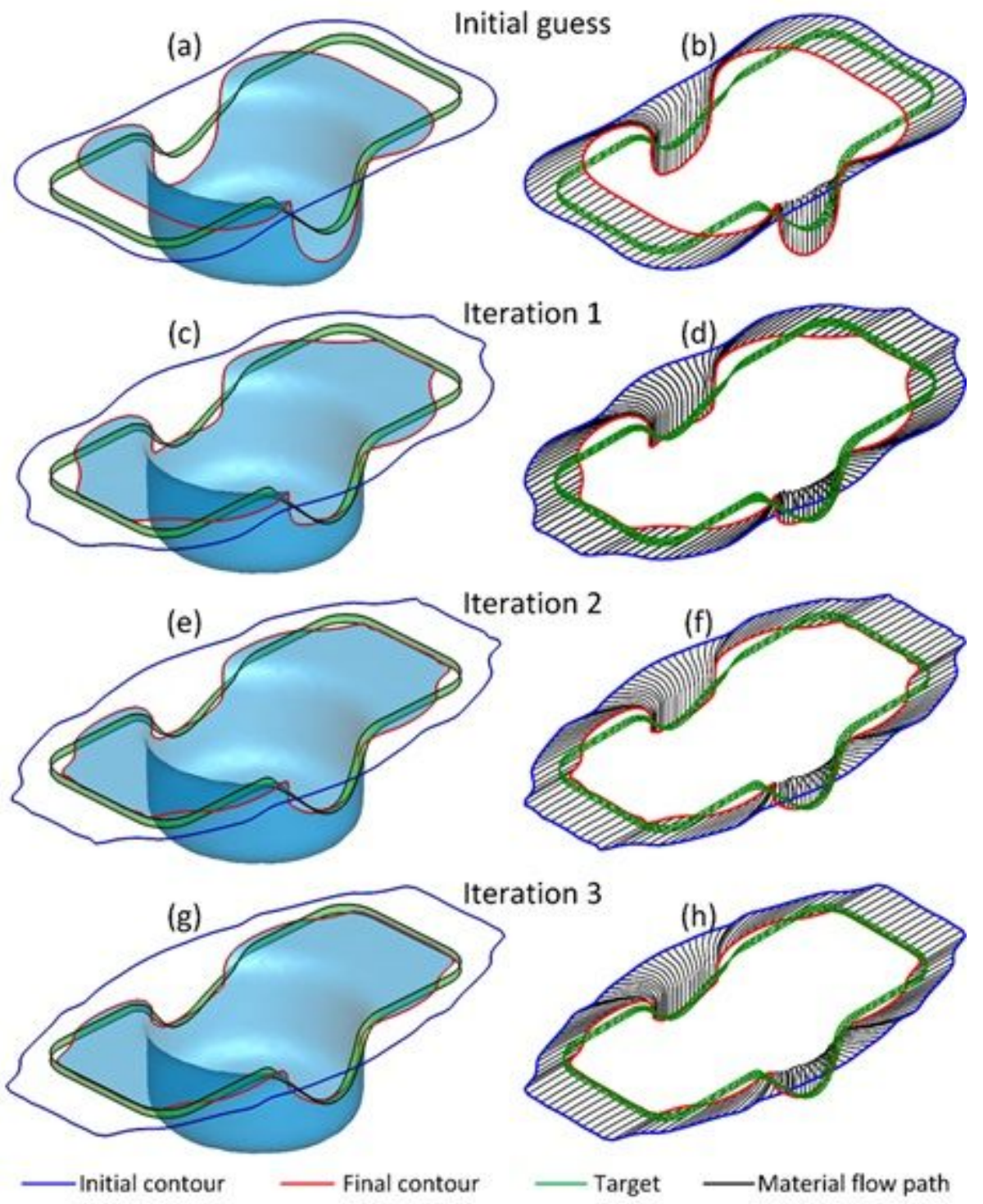

Figure 15

Results of example 3; (a) the initial guess for the blank; (b) The material flow paths of the boundary points; (c) The modified initial blank and the deformed plate; (d) material flow path of the boundary points; (e) to (h) The second and third iterations. 


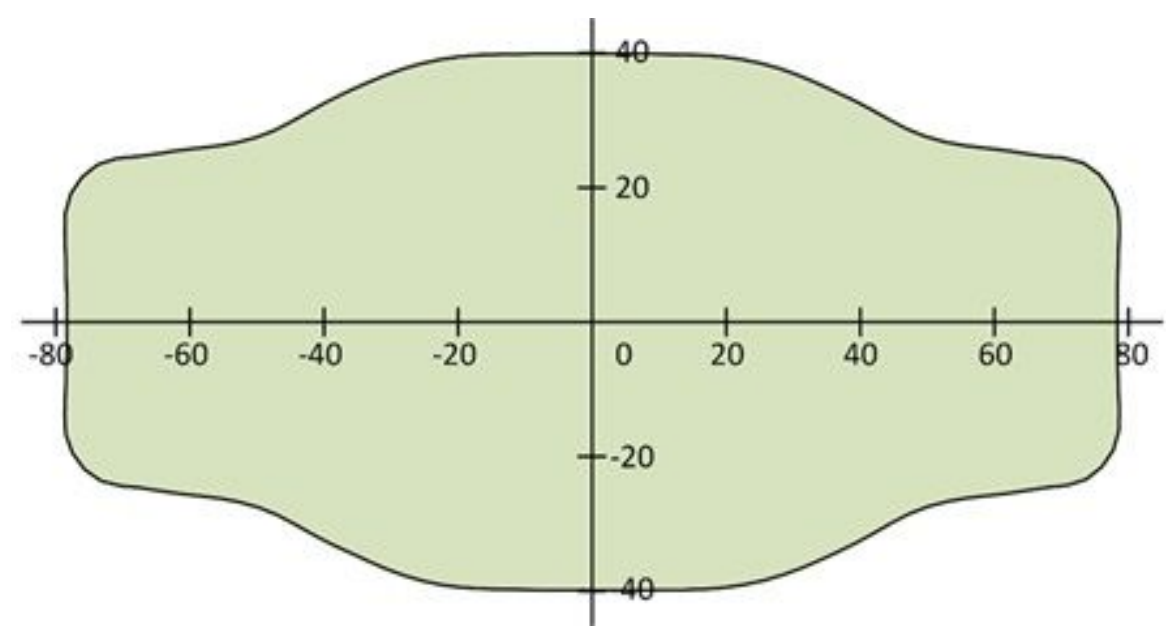

Figure 16

The optimum initial blank for example 3 .

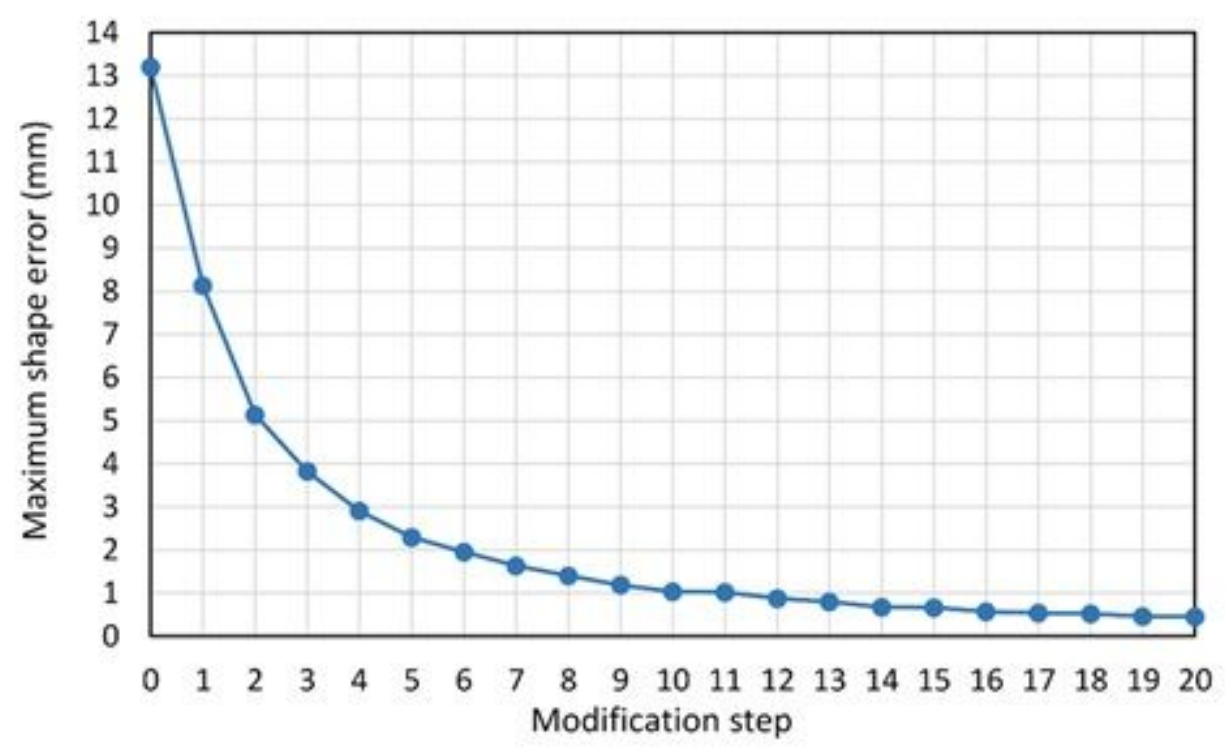

Figure 17

The maximum shape error for different iterations for example 3. 
(a)
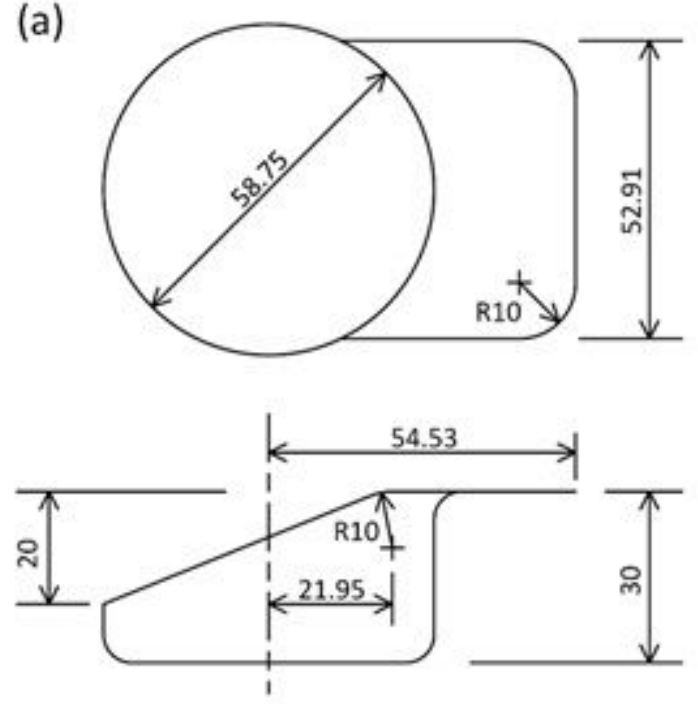
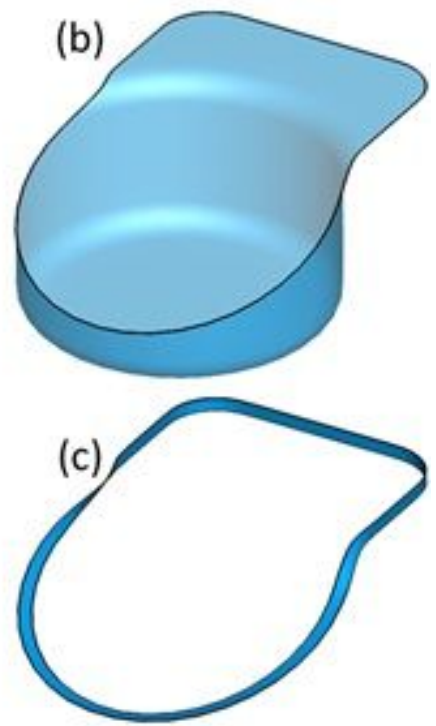

Figure 18

The geometric details of the target shape for example 4; (a) The front and top view of the target final product; (b) The 3D view of the target product; (c) The target ribbon.
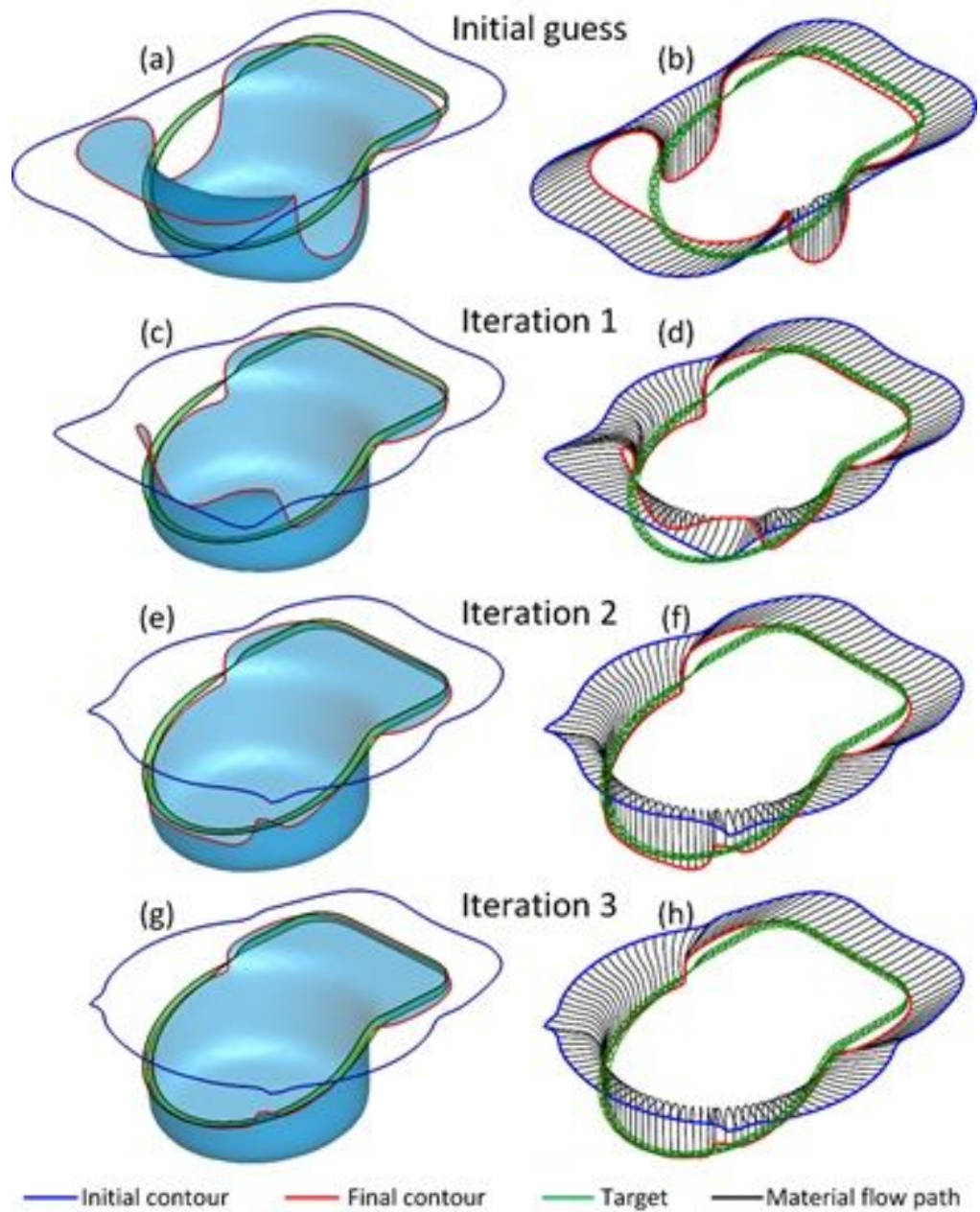

Figure 19 
First three steps for example 4; (a) The initial guess for the blank and the deformed plate; (b) The material flow paths of the boundary points; (c) The modified initial blank and the deformed plate; (d) The material flow path of the boundary points; (e) to (h) The second and third iterations.

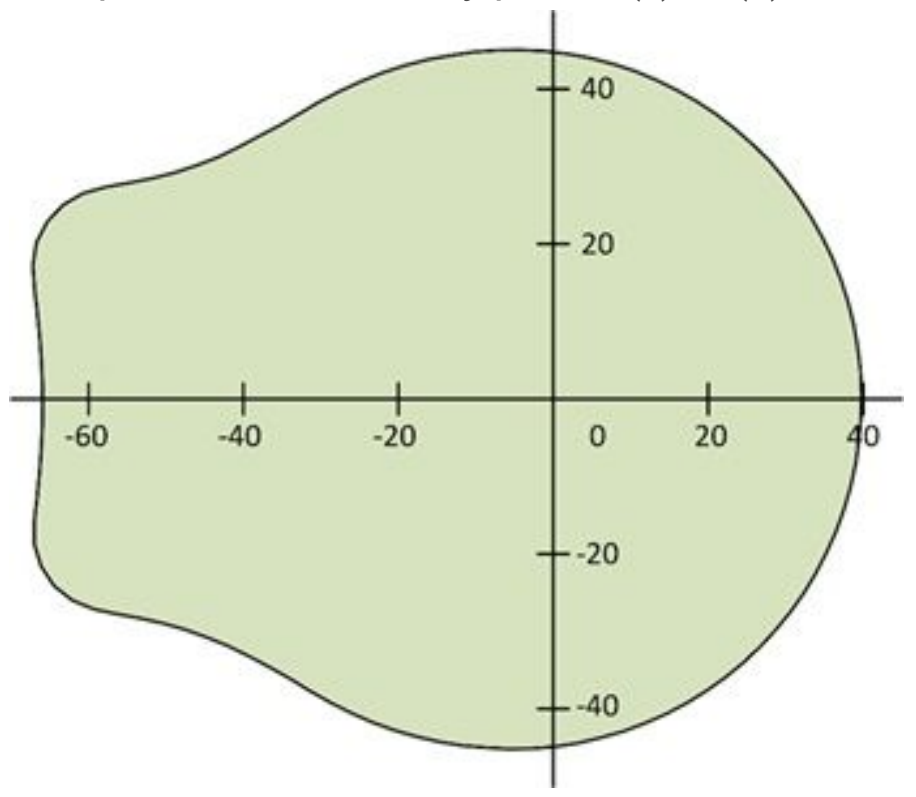

Figure 20

The optimum initial blank for example 4 .

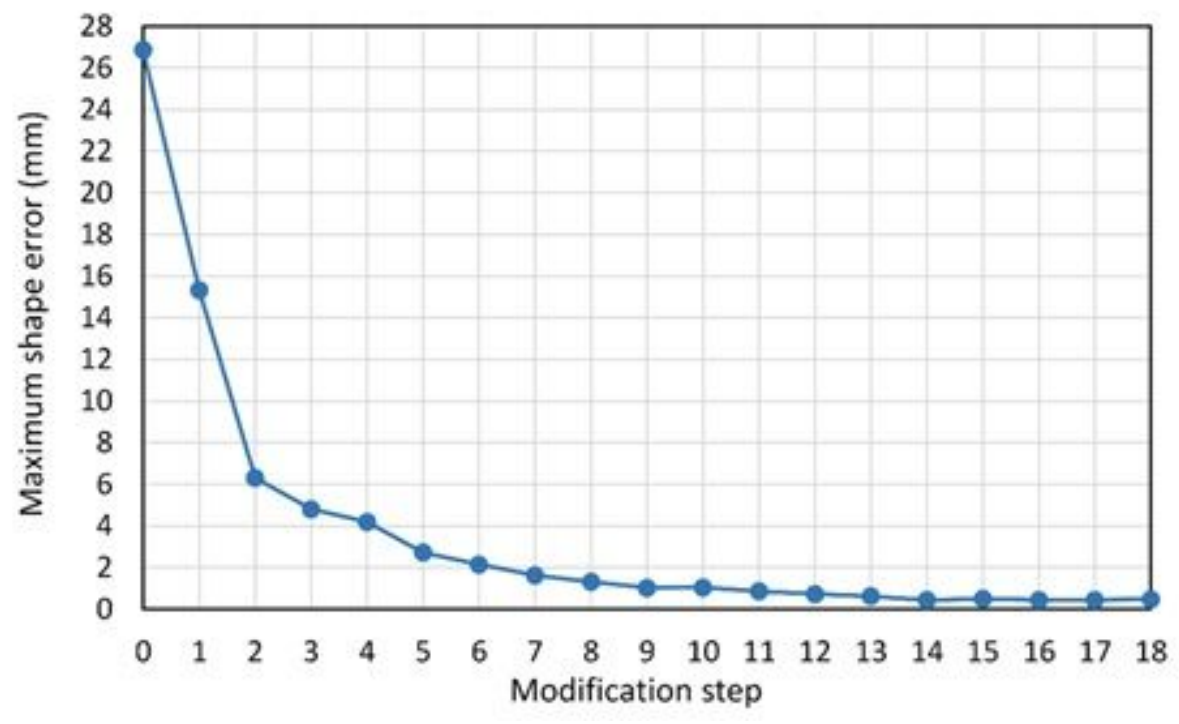

\section{Figure 21}

The maximum shape error for different iterations for example 4 

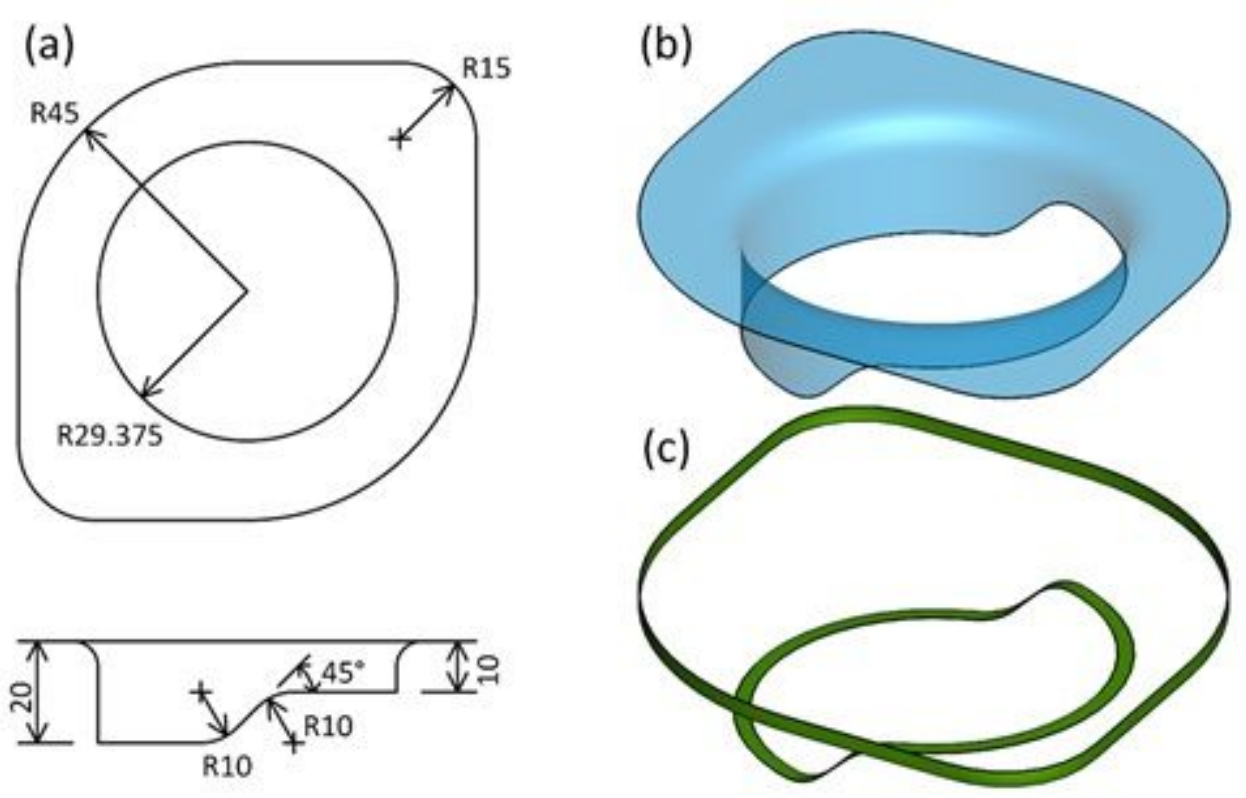

Figure 22

The geometric details of the target shape and internal and external targets for example 5; (a) The front and top view of the target final product; (b) The 3D view of the target product; (c) The target ribbons for the internal and external targets. 

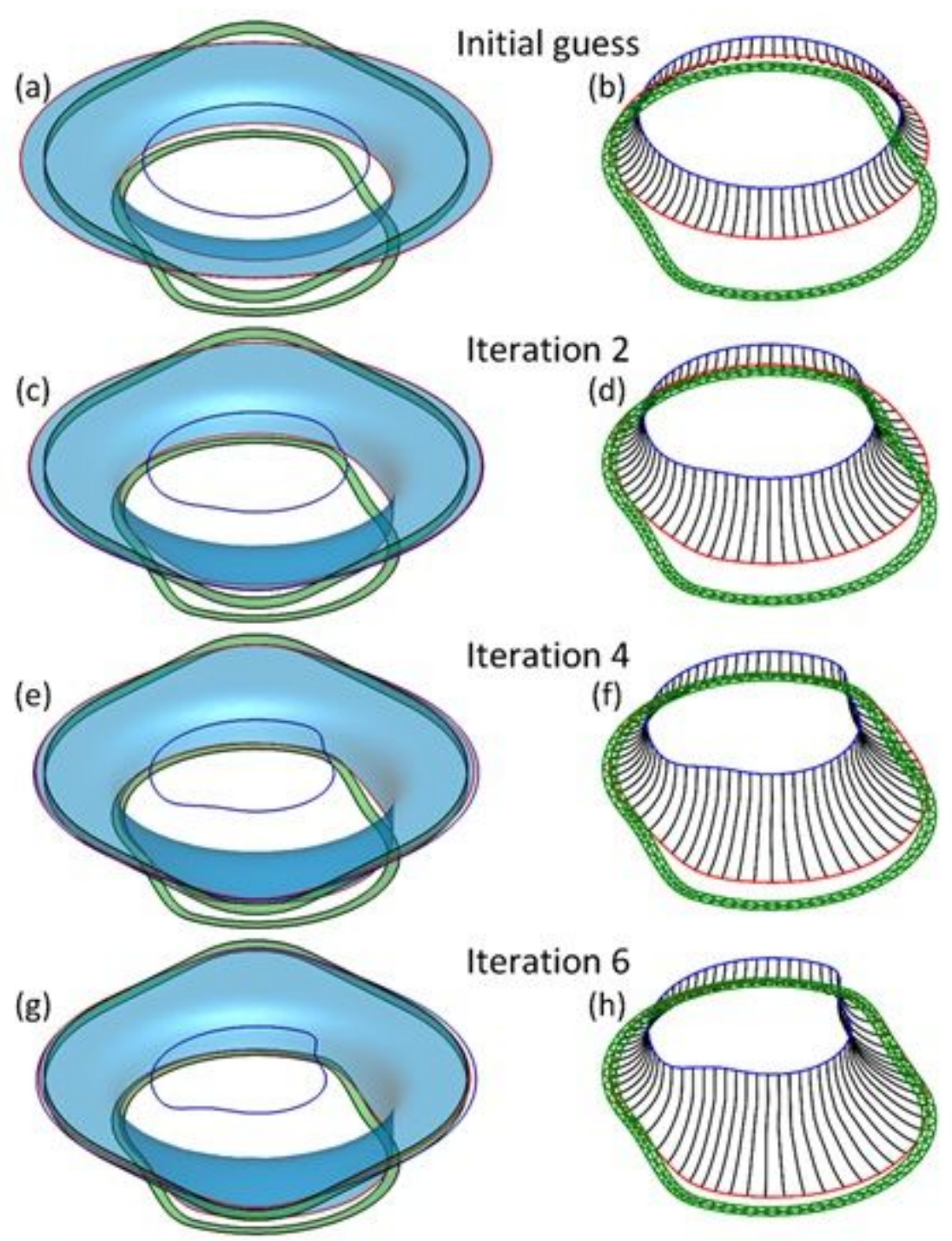

—Initial contour - Final contour —Target -Material flow path

\section{Figure 23}

Results of example 5 (three iterations 2, 4 and 6); (a) The initial guess for the blank and the deformed plate; (b) The material flow paths of the boundary points; (c) The modified initial blank and the deformed plate; (d) The material flow path of the boundary points; (e) to (h) The fourth and sixth iterations. 


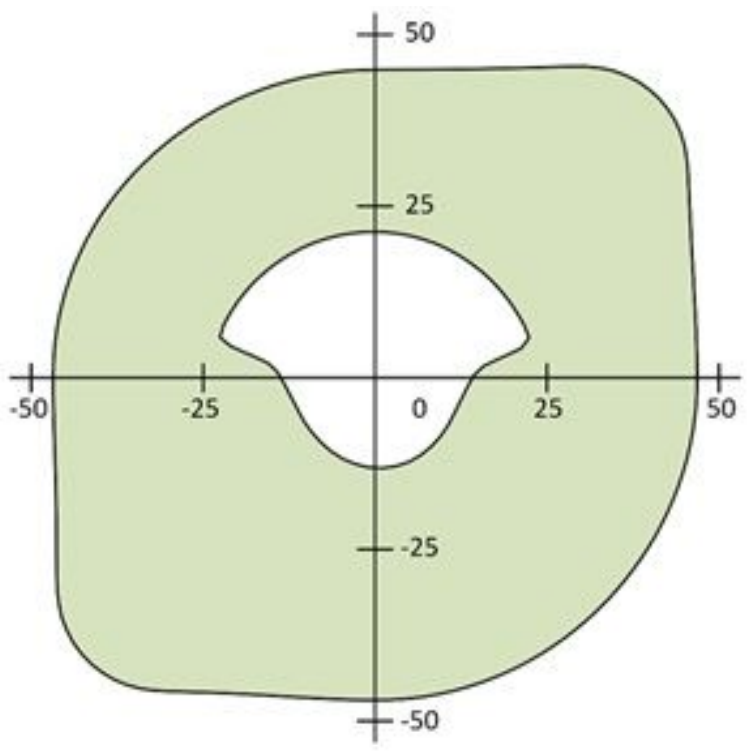

Figure 24

The optimum initial blank for example 5 .

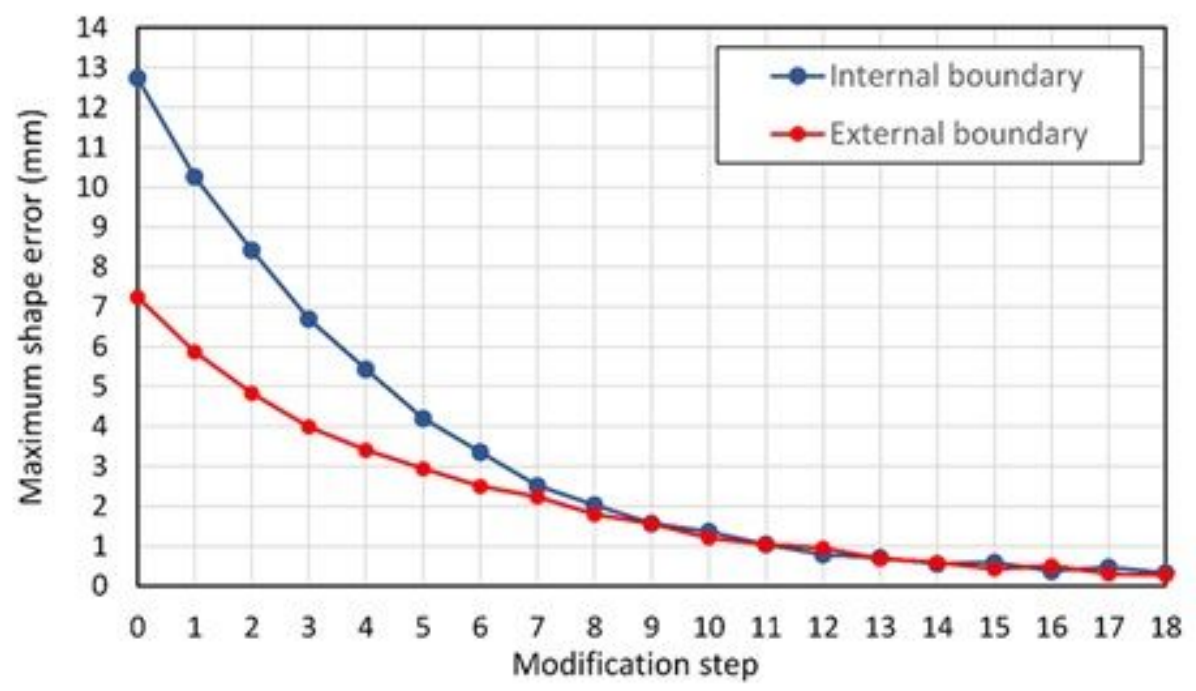

Figure 25

The maximum shape error for different iterations for example 5. 